مستقبل الخدمات المصرفية الإليكترونية

بين المخاطر وتحقيق الربحية

دراسة تطبيقية على الواقع الجديد للجهاز المصرفي المصري

د/ محمد غنيمى شندى إبراهيم

أكاديمية الإسكندرية للإدارة والمحاسبة 
الملخص

في ظل التحديات الاقتصادية التي تحملها رياح القرن الجديد ومنها العولمة المصرفية والتكتلات الاقتصادية الكبرى بين العديد من دول العالم كان لا بد أن ثتوائم الخدمات المالية مع مستحدثات العصر ، حتى يمكن أن تكون بالسرعة والدقة المطلوبة على المستوى الدولي ، حيث نجد أن أدوات التكنولوجيا المتقدمة قد دخلت بصورة مكثفة في القطاع المصرفي في الفترة السابقة ، وقد أثتتت كفاءة وفعالية كبيرة في الأداء ومسايرتها الخدمات المصرفية بشكل كبير وانعكس ذلك على نمو وسائل الدفع الاليكترونية وتطوير الخدمات المصرفية بسرعة وفعالية . وتعد تطبيقات الصيرفة الاليكترونية من المجالات المستحدثة والتي طرقتها العديد من البنوك المصرية و التي يتوقع أن يتسع نطاق التعامل بها مستقبلاً في ظل التقدم المتسارع الذي تشهده صناعة تكنولوجيا المعلومات في مصر من جهة ، وتز ايد أعداد مستخدمي شبكة الانترنت بصورة عامة من جهة أخرى و لا شك أن تطبيق أنظمة البنوك الاليكترونية يستلزم تو افر العديد من الركائز الأساسية سواء ما كان منها تجهيزي مثل توفير البنية الأساسية للمعلومات والاتصالات ، أو على المستوى البشرى و المتمثل في تو افر الكو ادر البشرية المناسبة والمؤهلة التأهيل الكافي ، بالإضافة إلى دعم الوعي المصرفي لاى المجتمع ، وتوعية العملاء بالمزايا التي يمكن أن تعود عليهم من جراء استخدام تلك الخدمات المستحدثة . 
مقدمة [ Introduction] [ [احب التطورات التكنولوجية تقدم مذهل في مجال الاتصالات و المعلومات ومعالجة البيانات ، حيث ساهمت في تطوير الأعمال والخدمات المصرفية سواء على المستوى المحلى أو الدولي الأمر الذي دفع البنوك إلى أداء خدمات مصرفية ومالية لم تكن بها من قبل فيل من خلال بناء شبكات واسعة من الفروع دون الاعتماد على أعداد كبيرة من العاملين ، وهو ما تحقق عن

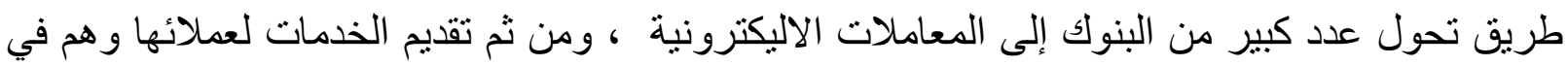

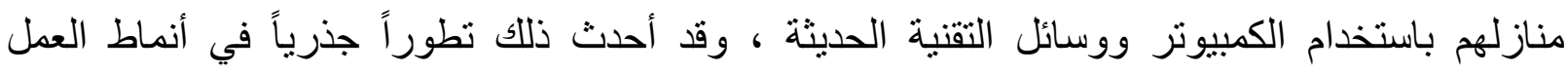

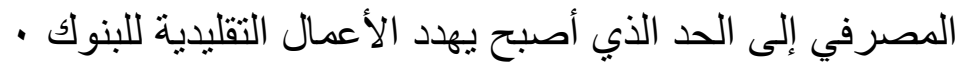

مشكلة البحث : تتمثل مشكلة البحث في كيفية معالجة الفجوة في الوضع الحالي بين التحديات التي تواجه الصيرفة الاليكترونية ممثلة في الكم الهائل من المخاطر التي تعوق تقديم الخدمات المصرفية الاليكترونية، وبين مدى إمكانية تحقيق الربحية في ظل هذه المخاطر - الأمر الذي يدفع باتجاه مدارسة تللك المشكلة بغية طرح بعض النتائج والتوصيات التي تعين المصارف على دلى دعم خطط وبرامج إدارة

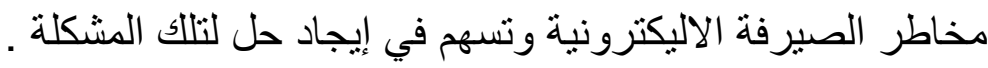
ويأتي ذلك تدعيماً للجهود المصرفية الدولية في ظل التحولات الاتتصادية العالمية ـ ومعايير لجنة بازل لتطوير الرقابة المصرفية ، وتقليل مخاطر الايتمان المصرفي أهداف البحث [ The Target of Research ]:يهدف البحث إلى التعرف على : أ_الخدمات المصرفية الاليكترونية أو ما ا أصطلح على تسميته بالصيرفة الاليكترونية . ب-مخاطر الخدمات المصرفية الاليكترونية التي لوحظ تعاظمها خاصة في السنوات القليلة الماضية

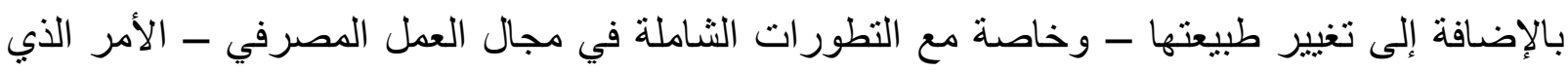

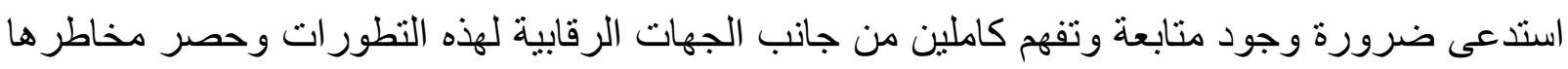
الرئيسية .

ج- مدى تحقيق الربحية في ظل تز ايد مخاطر الصيرفة الاليكترونية .

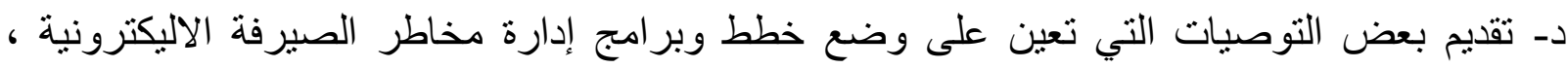

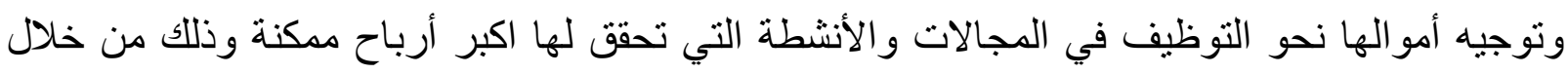
تحقيق عو ائد تزيد في مقدارها عن قيمة الفو ائد التي يتم دفعها للمودعين - كما تكفى لتغطية كل التكاليف

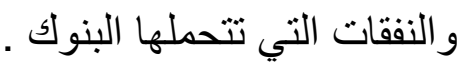


أهمية البحث [ The Importance of Research ] : من خلال استعر اضنا للأهداف السابقة تتضح بعض جو انب أهمية هذا البحث حيث يسهم في :أ- بلورة مفهوم الصيرفة الاليكترونية بما تمثلك من

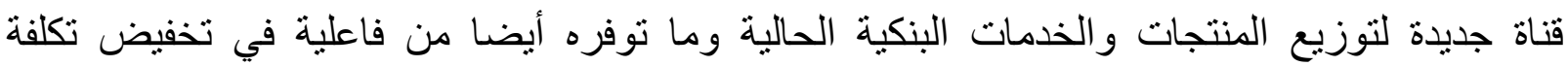
المعاملات و إيجاد سبل جديدة لجذب المزيد من العملاء الجدد فهي توفر على العميل زيارته للبنك لأداء

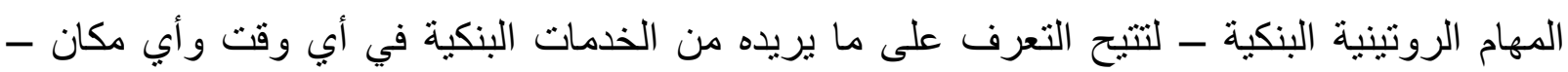

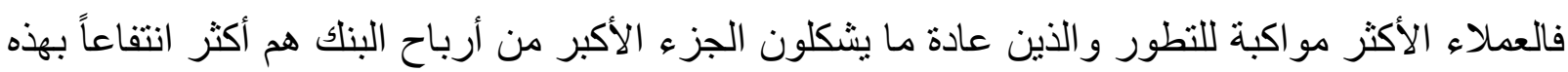

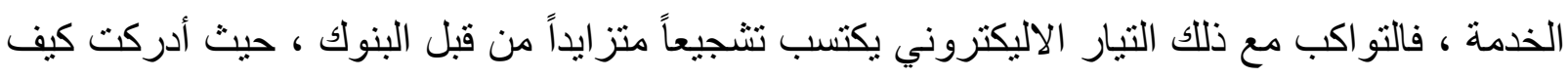

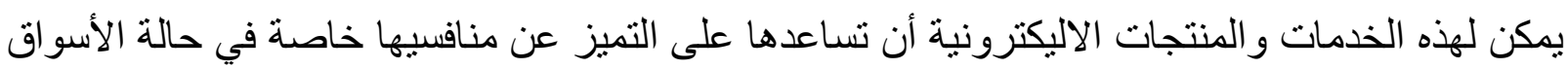

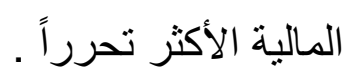
ب-كذللك يمكن القول بأن أهمية البحث تمتد التشمل بيان أن التحدي الاليكتروني يكمن فيما هو أكثر من

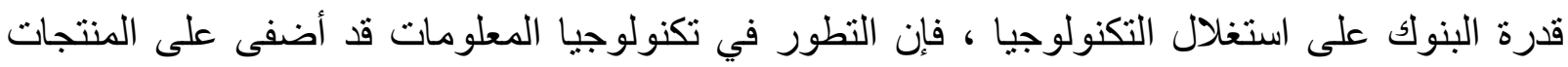

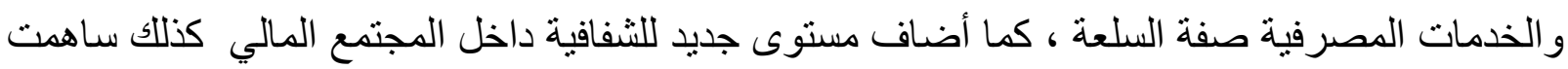
الثبكات الاليكترونية في الكثف عن الاختلافات السعرية ، وأعطت العملاء مساحة غير محدودة جغر افيا للوصول لأكبر عدد من المنافسين ، فحالياً أصبح في استطاعة العملاء مستخدمي شبكة الانترنيت مقارنة الأسعار والمنتجات بسهولة ، وبالتالي يوجد تحدى جديد أمام البنوك.

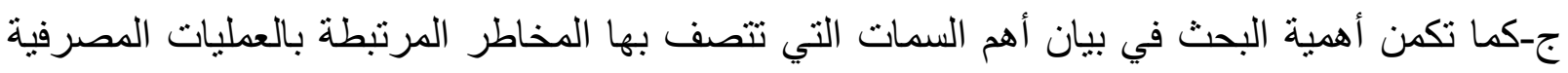
الاليكترونية والتي منها التغير السريع في الابتكارات التقنية والخدمات المصرفية التهية المصاحبة واستخدام

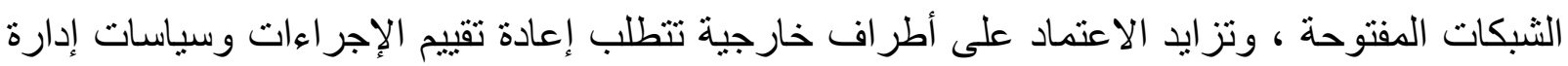
المخاطر بشكل مستمر على ضوء التبدلات في تكنولوجيا المخاطر المصرفية التي تعزز هذه العمليات . د- بيان اهتمام المصارف والسلطات الرقابية بالعلاقة بين المخاطر و الربحية . منهج البحث ومحتوياته [ Methodology ] :وتشياً مع المنهجية العلمية في الأبحاث النظرية ـ ومن أجل تحقيق الأهداف المشار إليها آنفاً فإنه سيتم التعرض بقدر مناسب من التفاصيل لكل الموضو عات و القضايا التي وردت في سياق تلك الأهداف بحسب ترتيب ورودها حيث راعينا المنهج الاستقرائي و الترتيب المنطقي الذي يعين على تطوير البناء النظري لمفهوم الصيرفة الاليكترونية ، وتفهم طبيعة

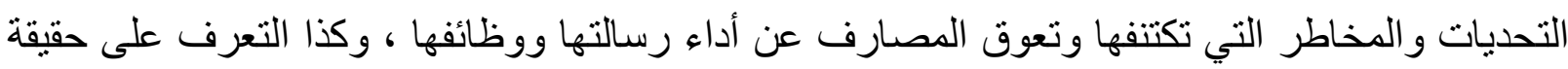

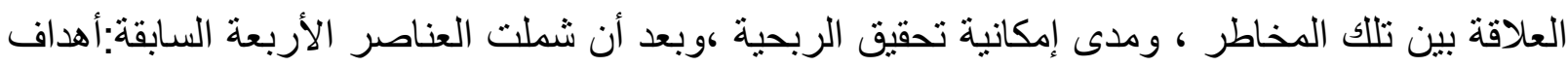


البحث و أهميته ، و مشكلته ، و المنهج المتبع فيه فإن العنصر الخامس ، سوف يخصص لإلقاء الضوء على مفهوم الخدمات المصرفية الاليكترونية وأنواعها، كما يشمل العنصر السادس مخاطر الصيرفة الاليكترونية وكيفية التعامل معها ، في حين يتعرض العنصر السابع لمدى إمكانية تحقيق الربحية في ظل هذه المخاطر المصرفية ، على أن يتبعه العنصر الثامن والأخير و الخاص بنتائج البحث وتوصياته وذلك على النحو التالي :

أولاً: الخدمات المصرفية الاليكثرونية [ مفهومها وأنواعها ] :

اـالتعريف بالخدمات المصرفية الاليكترونية : يقصد بالخدمات المصرفية الاليكترونية : تقديم البنوك الخدمات المصرفية التقليدية أو المبتكرة من خلال شبكات اتصال اليكترونية تقتصر صلاحية الدخول إليها على المشاركين فيها ، وفقاً لشروط العضوية التي تحددها البنوك وذلك من خلال أحد المنافذ على الثبكة كوسيلة لاتصال العملاء بها بهدف : أ- إتاحة معلومات عن الخدمات التي يؤديها البنك دون تقديم خدمات مصرفية على الثبكة . ب- حصول العملاء على خدمات محدودة كالتعرف على معاملاتهم وأرصدة حساباتهم ، وتحديث بياناتهم وطلب الحصول على قروض .

ج- طلب العملاء تنفيذ عمليات مصرفية مثل تحويل الأموال ،ولأغر اض هذه الضوابط فإن البنوك التي تقوم بتقديم الخدمات الواردة بالبند [ ج ] فقط تعتبر بنوكاً تقدم عمليات مصرفية اليكترونية تتطلب نوافر سياسات و إجر اءات لتقييم المخاطر Assessing و الرقابة عليها Controlling ومتابعتها إلا أنه يجب أيضاً على البنوك مر اعاة الإدارة الحصيفة لائية مخاطر بشأن العمليات الواردة بالبندين [ أ ] - $[$ و

ץ- أنواع الخدمات المصرفية الاليكترونية (' ) : فيما يتعلق بأنو اع الخدمات المصرفية التي توفرها البنوك الاليكترونية في الوقت الحاضر فهي على النحو التالي :

1-Bernardo Batiz -Lazo ,A historical Appraisal of information Technology in commercial Banking University Business School, july 2002 , p2

- Richard Li Hua ,(2007)Bench marking china firm competitiveness :a strategic framework, journal of technology management in china, volume :2,issue:2.(on- line) available - $\quad$ on:www.emeraldinsight.com , 21/4/2008 
أ- بطاقات الدفع الاليكترونية ( ): هي أداة مصرفية للوفاء بالالتزامات تجد قبو لاً على نطاق واسع في

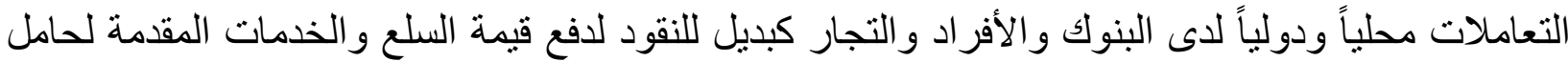
البطاقة ، ويوجد نوعين أساسيين من بطاقات الدفع : - بطاقات الخصم [ Debit Cards ] ويقتصر استخدامها خصما على حسابات دائنة للعملاء ويتوقف استخدام البطاقة على رصيد حساب العميل لدى البنك ،حيث يتم رفض البطاقة إذا تجاوز مبلغ العملية رصيد

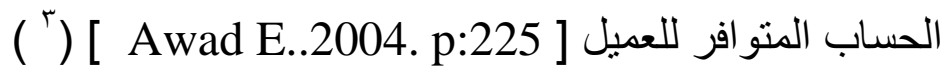

- بطاقة السداد الثهرى : [ Charge Card ] تمنح هذه البطاقة حاملها ائتماناً شهرياً غير متجدد من قبل البنك مصدر البطاقة ، وبنهاية كل شهر يقيد إجمالى السحوبات على حساب العميل حامل البطاقة ويلتزم

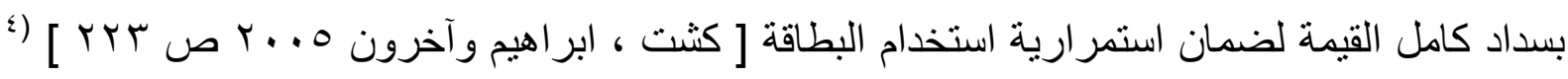

- بطاقات الايتمان [ Credit Cards ] ويتم استخدامها خصماً على حسابات مدينة وفقا للحدود المقررة وتستخدم الوحدات الطرفية لنقاط البيع Point of Sale Terminals وآلات الصرف الآلي Automatic Teller Machines

$$
\text { الإليكترونية (" ) }
$$

بـ الخدمات المصرفية عن بعد [ Remote Banking Services ] وهى الخدمات التي تتم من خلال قنوات التوزيع الاليكترونية التي ينشرها البنك خارج نطاق حيزه المكاني كماكينات الصيرفة الآلية

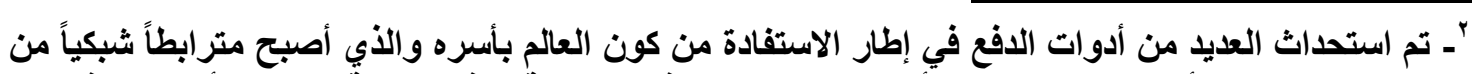

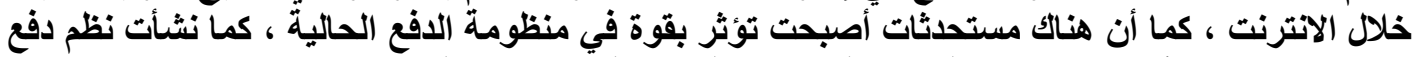

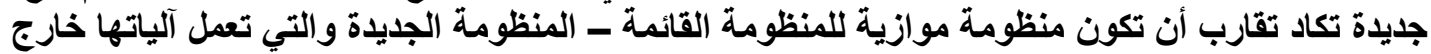

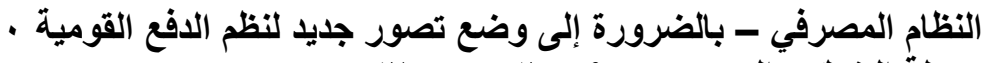

3-Awad Elias, (2004) Electronic Commerce: From Vision to Fulfillment ,Pearson Prentice Hall, Upper Saddle River New Jersey

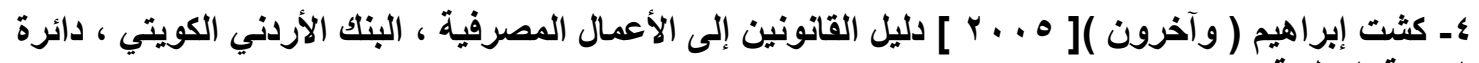

$$
\begin{aligned}
& \text { المكتبة الوطنية ،عمان . أنرون }
\end{aligned}
$$

5-Electronic cash : The Funds or value is stored on an electronic device as the personal computer of the consumer Which is loaded using specialized software EC is used to Make small payments through a transfer of value to the merchants electronic device 
ATMS وماكينات منح القروض الآلية ALMS ونقاط البيع الاليكترونية EPOS ، حيث يقدم عن طريقها كافة الخدمات المصرفية [ المالية وغير المالية ] التي يحتاجها العملاء باستخدام بطاقات الدفع الاليكترونية

.( ) [ Turban .and Vihland .2004-p099] ج- خدمات الصيرفة المنزلية [ Home Banking] وهى من الخدمات المستحدثة نسبياً وتشمل كافة الخدمات المصرفية [ المالية وغير المالية ] كالاستفسار عن الأرصدة وطلب كشوف حسابات ، وبعض الخدمات المالية كدفع الفواتير والتحويل بين حسابات العميل أو إلى حسابات عميل آخر ، ويمكن للعملاء

Phone Banking - Mobile Banking - Home : الحصول على تلك الخدمات عن طريق

Banking

د- الخدمات المصرفية عبر شبكة الانترنيت Internet : وهى تعد من أهم قنوات البنوك الاليكترونية ، وتثمل الخدمات المصرفية المالية وغير المالية من استفسارات عن المنتجات و الخدمات التي يقدمها البنك وكيفية الحصول عليها ، و أيضاً الإعلان عن أسعار الصرف وأسعار الفائدة بالبنك ودفع فواتير الخدمات وإجراء التحويلات المالية وفتح الحسابات ، علاوة على الخدمات المحلية مثل دفع الفو اتير الاليكترونية [ Kotler and Armstrong. 2004 . ( ) . وتنثير الدراسات في هذا المجال إلى نحو • ^ \% من إجمالي البنوك على مستوى العالم تمنلك مواقع لها على شبكة الانترنيت ، بل أن جميع البنوك الأمريكية يكاد يكون لها مواقع على تلك أل الثبكة [^ ] ، وقد نتج عن ذلك أن تز ايد عدد البنوك التي تتصف معاملاتها بالسرية على صفحات

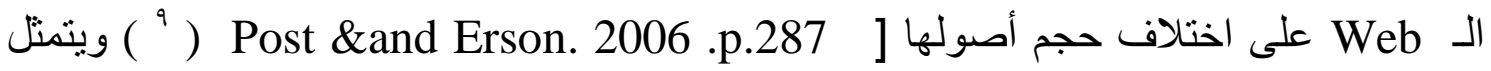

6 Turban \& Viehland, (2004) Electronic Commerce " A managerial Perspective Pearson Education , tnc., Upper Saddle, River, New Jersey

7-Kotler ,PH . and Keller, K.L, (2006) Marketing Management "Pearson Prentice Hall Upper Saddle River, New Jersey

8- الأكاديمية العربية للعلوم المالية والمصرفية ، مجلة الدراسات المالية والمصرفية ، المجلا الثامن ، العدد

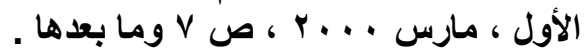

9- Kotler,PH.And Armstrong ,G (2004)"Principle of Marketing "Pearson Prentice Hall, Upper Saddle River, New Jersey 
الجانب الآخر الهام في تقديم الخدمات المصرفية عبر شبكة الانترنيت في تأسيس ما يعرف بالبنك

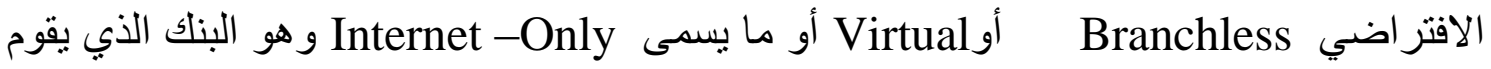
بتقديم كافة العمليات المصرفية من سحب وإيداع من خلال ماكينات الصسارف الآلي ATMS أو من خلال قنوات التوزيع عن بعد Remote Delivery Channels التي تملكها المؤسسات

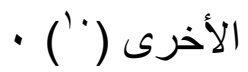

هـ- الخدمة المصرفية عبر الهاتف النقال [ WAP ] تعتبر هذه الخدمة من أحدث التقنيات التى تقدمها البنوك ، و هى تتيح لعملاء البنك الوصول إلى حساباتهم المصرفية و إجراء معاملاتهم عبر اجهزة الهاتف الخلوى بيسر وأمان ، فى أى مكان و على مدار ع ساعة ، كما تتيح لهم المجال للاستفادة من خدمات عديدة مثل : التحويل المالى بين حسابات داخل البنك ، التحويل المالى إلى عميل آخر فى البنك ، تسديد الفواتير ، معرفة العمليات التى تمت على الحساب ، الاستفسار عن أرصدة حساباتهم الجارية ، والتوفير والودائع ، الحصول على كثف حساب ملخص ، طلب دفاتر شيكات ، وطلب بطاقة ائتمان ، إضافة إلى الخدمات التى تقدمها المصارف الاليكترونية من خلال خدمة الرسائل القصيرة [ SMS ] ، خدمة الصر اف الآلى ، وخدمة البنك الناطق من خلال هاتف البنك المصرفى [ Joseph .p.t.2004.p:290 J'") ، كثت ، ابر اهيم

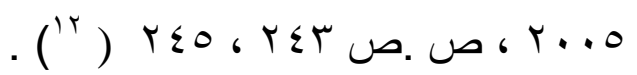
rـ البنولك المصرية وإصدار بطاقات الدفع الاليكترونية (r") :تعمل البنوك المصرية جاهدة على اللحاق بركب التكنولوجيا الحديثة ، والعمل على تحقيق الاستفادة المرجوة من ثورة الاتصالات والمعلومات والانفتاح على الأسواق الخارجية ، خاصة مع التنفيذ الفعلي لاتفاقية تحرير التجارة في الخدمات GATS وذلك في إطار الاتفاقية العامة للتجارة والتعريفات GATT و انضمام مصر - في يونيو r + . - ـ لعضوية اتفاقية الاتصالات التابعة لمنظمة التجارة العالمية

10--Karen Furst .William W.Lang.and Daniel E.Nolle. Internet Banking : Developments and Prospects.Economic And Policy Analysis Working Paper 2002 9 September 2000.p16

11-joseph ,P.T, (2004) E.commerce;;Amanagerial Perspective "Prentice Hall of India ,Private Limited, New Delhi

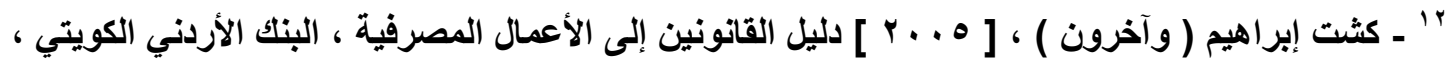

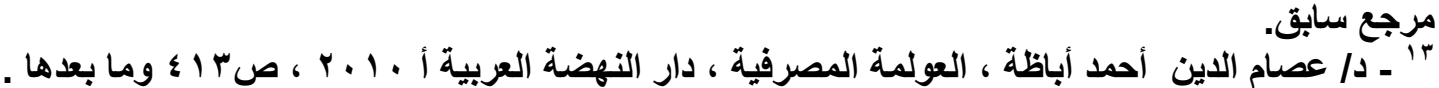


[ BAT [ Basic Telecommunication Agreement إلى الدخول بقوة إلى أعمال البنوك الاليكترونية على اختلاف خدماتها ، وفى هذا النطاق يقوم نحو

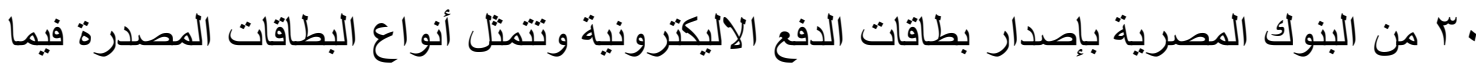
أ-بطاقات الايتمان Credit Cards تقوم البنوك المصرية بإصدار بطاقات ائتمان دولية بالتعاون

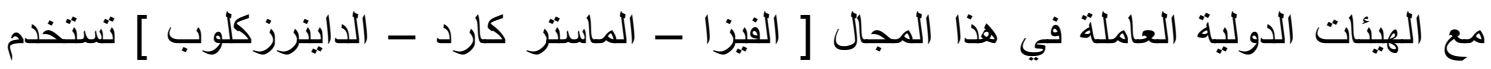

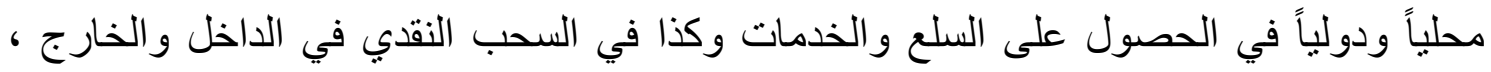

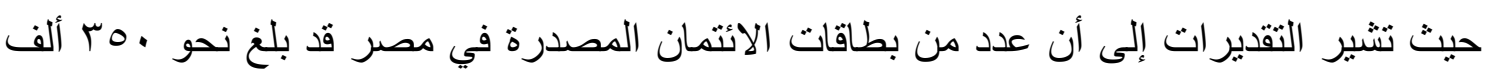

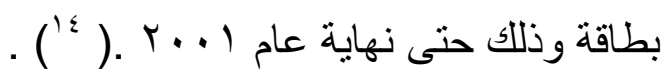
ب- بطاقات الخصم Debit Cards : تصدر البنوك المصرية في الوقت الحالي لعملاء الفروع من أصحاب الحسابات الجارية وحسابات التوفير بطاقات صرف آلي ATM تستخدم في السحب

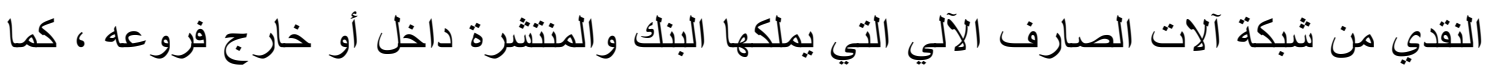
تتجه بعض البنوك المصرية في الوقت الحالي إلى إصدار بطاقات الخصم الدولية ، وذلك لتحويل

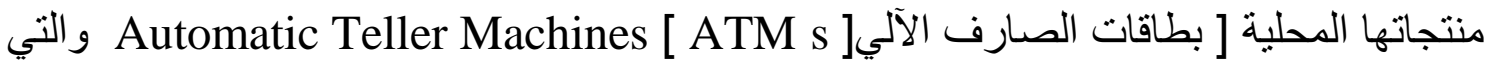
تستخدم داخل شبكة البنك المصدر فقط إلى بطاقات خصم دولية [ إلكترون - ماستر ] بحيث يمكن

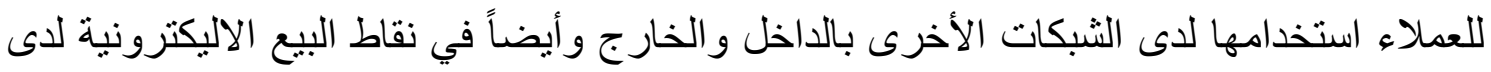
المنشآت التجارية ، ومن ثم أصبحت المنافسة بين البنوك على الخدمات التي تقدها البطاقات

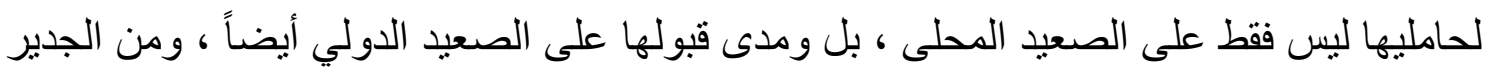

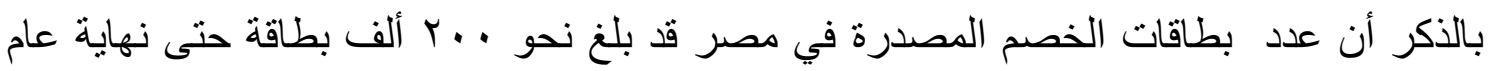
- $\left({ }^{10}\right)$ Y... ع- دور البنوك المصرية في مجال نشر قنوات التوزيع الاليكترونية : تقوم البنوك المصرية بالتوازي مع التوسع في إصدار بطاقات الدفع الاليكترونية بالتوسع في نشر قنوات

14-American Chamber of Commererce in Egypt Information Technology in Egypt , April 2002 , p111- 112

ا ـ البنك الأهلي المصري ، النشرة الاقتصادية ، تطبيقات الصيرفة الاليكترونية في مصر ،العدد الثالث ، المجلد 
التوزيع الاليكترونية لخلق سوق مناسب لقبول البطاقات داخل مصر حتى يتحقق للنظام النجاح المطلوب وفيما يلي أهم قنوات التوزيع الاليكترونية التي تعتمد عليها البنوك المصرية : أـ نقاط البيع الاليكترونية ] Electronic Point Of Sale [ EPOS : تقوم بعض البنوك المصرية بنشر شبكة من نقاط البيع الاليكترونية EPOS لدى المنشآت التجارية بمختلف أنشطتها بجميع مناطق الجمهورية - تقدر بنحو V لآلاف نقطة [ تاجر ] وذلك لتسهيل الخدمات اللازمة لمستخدمي البطاقات المحلية والدولية ، كما توفر البنوك داخل فروعها خدمة السحب النقدي لحاملي بطاقات الدفع الاليكترونية المحلية والدولية ، كما تقبل بعض البنوك الإيداعات النقدية لحاملي البطاقات الصادرة عن طريقها من خلال آلات ال EPOS بـ آلات الصارف الآلي :Automatic Teller Machines بدأت البنوك المصرية في تقديم خدمة ال ATMS في الثمانينات من القرن الماضي وكان استخدام البطاقات آنذاك يقتصر على الآلة الخاصة بكل فرع مصدر فقط ، ثم أصبح من الممكن استخدام البطاقة من خلال شبكة ماكينات البنك الو احد اعتبار اً من عام 991 19 ، كما ارتبطت بعض البنوك المصرية باتفاقيات تتيح استخدام البطاقات الصادرة من أي منها في ماكينات الآخر ، ويقوم عدد • سبنكاً من البنوك المصرية [ ذات البنوك المصدرة للبطاقات ] بنشر نحو . . . ماكينة صرف آلي ATMS ثم تركيبها ونشر ها داخل وخارج الفروع [ بالإضافة إلى نحو . 1 آلة أخرى تحت التجهيز ] لتقديم خدماتها لجميع حاملي بطاقات الدفع الاليكترونية الصادرة من البنوك المحلية والعالمية بنوعيها بطاقات الائتمان وبطاقات الخصم والتي تحمل العلامات المميزة للهيئات الدولية المختلفة [ الفيزا ـ الماستر كارد - الداينرزكلوب] ( 17$)^{17}$ وتتيح البنوك المصرية العديد من الخدمات التي تقدمها آلات الصرف الآلي ATMS الخاصة بها ، والتي تختلف درجة تطبيقها من بنك لآخر ، وفقاً لاعتبارات عديدة لعل من أهمها الرغبة الصادقة لإدارات البنوك في التحول نحو الصيرفة الاليكترونية ، ومدى توافر الموارد المالية والكوادر الفنية المدربة على استيعاب التكنولوجيا الحديثة وكذا تو افر معايير الرقابة الاليكترونية الحصيفة ومن أهم الخدمات المتاحة في هذا المجال

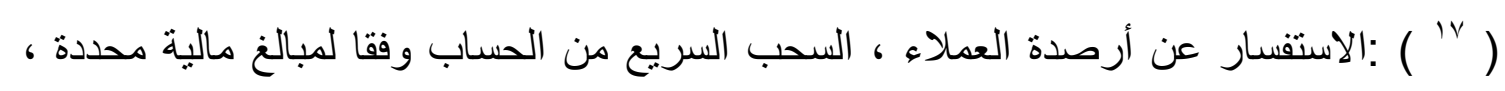

'" ـ البنك الأهلي المصري ، النشرة الاقتصادية ، تطبيقات الصيرفة الاليكترونية في مصر ، العدد الثالث ،

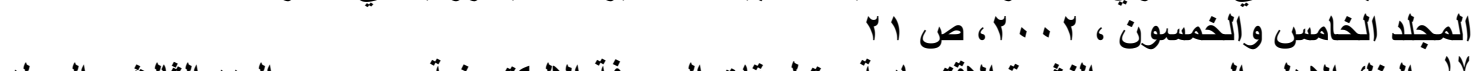
Vا ـالبنك الاهلى المصري ، النشرة الأبتصادية ، تطبيقات الصيرفة الاليكترونية ى مصر ، العدد الثالث ، المجلد 
السحب بالمبالغ التي يحددها العملاء والتي يسمح بها حسابهر ، طلب كثف حساب ، إيداع النقد والثيكات ، طلب دفتر شيكات ، إمكانية تغيير الرقم السري لبطاقات الصارف الآلي ، التحول بين حسابات العميل الواحد ، خدمة دفع فو اتير الخدمات [ التليفونات -كهرباء ـغاز - مياه ] خدمة الاستفسار عن أسعار الخدمات المصرفية ، دفع مستحقات بطاقات الائتمان [ الفيزا و الماستر كارد

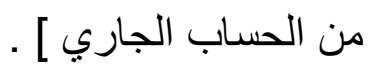
ج-- البنك الثخصي [ الصيرفة المنزلية ] Home Banking إستكمالاً لمسايرة الفكر العالمي المتطور في مجال خدمات البنك الشخصي الذي بعتمد على أحدث النظم التكنولوجية ، والذي تعتمد عليه البنوك العالمية الكبيرة ذات العدد الضخم من العملاء ، فقد انتهت بعض البنوك المصرية من تركيب وتشغيل مر اكز للاتصالات وخدمة العملاء Call Center وذلك بدءً من عام 999 ، ، حيث تتيح الخدمة المصرفية تليفونياً لعملائها باستخدام رقم تمييز شخصي ، وتقوم الخدمة بتلقي استفسار ات العملاء وطلباتهم من خلال التليفون على مدار ع باعة يومياً والرد

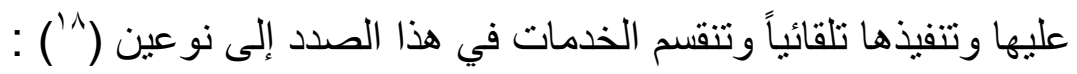
النوع الأول: خدمات لا تتضمن إجراء عمليات مالية وتشمل : الإستعلام عن أرصدة بطاقات الائتمان ، الاستعلام عن آخر حركة سداد ، الاستعلام عن آخر كثف حساب ، الاستعلام عن أرصدة الحسابات الجارية والحركات على الحساب ، استلام كثف حساب بالفاكس عن أي شهر من الثهور rا السابقة الاستعلام عن الخدمات المصرفية المتميزة التي يقدمها البنك ، الاستعلام عن أسعار صرف العملات الأجنبية ، الاستعلام عن أسعار الفوائد على الودائع . النوع الثاني : خدمات تتضمن إجراء عمليات مالية : وتنثمل إتاحة بعض الخدمات المالية لجميع حاملي البطاقات [ الائتمان - الخصم كالتحويلات بين حسابات العميل ودفع فو اتير بعض الخدمات د- البنك المحمول Mobile Banking : (19 ) بدأت بعض البنوك المصرية [ عدد r بنوك بدءً من عام . . . ب في تطبيق نظام البنك المحمول و الذي يسمح للعملاء باستخدام التليفون

ـ البنك الاهلى المصري ، مركز البطاقات ، البنوك الاليكترونية وتطبيقاتها في مصر دراسة غير منشورة ،

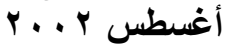

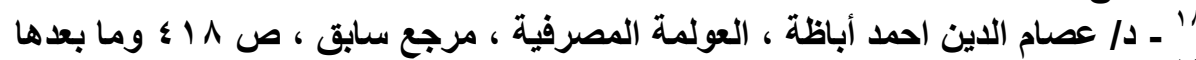

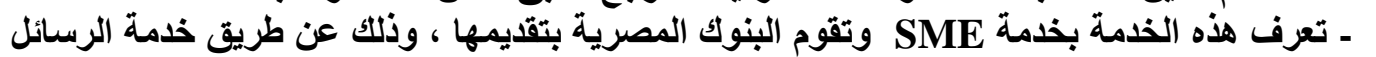

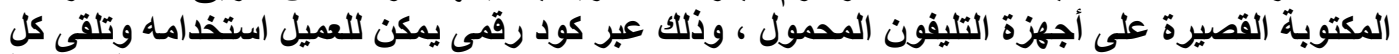

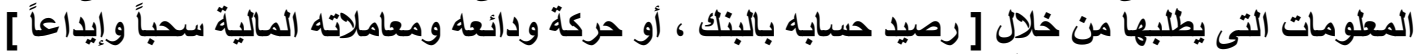

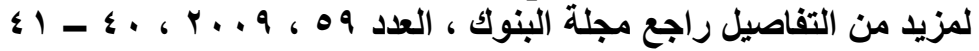


المحمول في الحصول على بعض الخدمات المصرفية باعتباره أحد قنوات توزيع تلك الخدمات لعملائها وذلك باستخدام أحدث تكنولوجيا الاتصالات المتطورة التي تملكها الثركات العاملة في هذا المجال والتي تمكنها من نقل جميع البيانات والمعلومات DATA بصورة مؤمنة ـويتم تقديم No - Financial الخدمة على النحو التالي الأول : خدمات لاتتضمن إجراء عمليات مالية Transaction ل للعملاء ممن لديهم تليفون محمول وتتيح لهم الاستعلام عن : رصيد حساب بطاقات الائتمان الفيزا - الماستر كارد ] ، الحد الأدنى المطلوب سداده قبل نهاية فترة السماح الحد المصرح به للبطاقة ،المتاح بالبطاقة أثناء الاستفسار ، آخر عدد 7 معاملات [ سحب - مشنريات ] تمت على البطاقة ، طلب كثف حساب . بـ تقديم الخدمات غير المرتبطة بحسابات العملاء و التي بطلق عليها Tele Marketing و التي تمكن العملاء من الحصول على المعلومات اللازمة عن المنتجات والخدمات التي يوفرها البنك وتشمل الاستعلام عن : المنتجات والخدمات التي يقدمها البنك ، أسعار صرف العملات الأجنبية ، أسعار الفوائد الدائنة على الودائع ،حسابات التوفير ،الأوعية الادخارية ،شهادات الاستثمار، موقع آلات الصارف الآلي ATMS المملوكة للبنك . الثاني : خدمات تتضمن إجراء عملية مالية Financial Transactions مرتبطة بحسابات العملاء من خلال منح العميل رقم سرى PIN لإدخاله على تليفونه المحمول وتشمل : التحويل من حساب بطاقة أخرى لنفس العميل بنفس الفرع ،الاستعلام عن فاتورة التليفون المحمول وسداد قيمتها خصماً على حساب بطاقات الفيزا ـالماستر كارد لعملاء البنك ،استخدام بطاقات الفيزا و الماستر كارد في عمليات إعادة شحن كارت التليفون المحمول ، [ نظام الكارت المدفوع مقدماً ] - M.Commerce عمليات البيع والثراء من خلال التليفون المحمول وتجدر الإشارة إلى أن هنالك العديد من الآراء التي ترى أن خدمة ال Mobile Banking تعد من أكثر استخدامات ال E- Banking أماناً نظراً لوجود رقم سرى خاص بالموبايل ، علاوة على وجود رقم سرى خاص بالعميل ، وبالنظر إلى ارتفاع عدد المشتركين في شبكتي التليفون

المحمول في مصر إلى نحو 9 .ب مليون مشترك ، فإنه يتوقع لهذه الخدمة أن تتوسع مستقبلاً . هـ الفرع الإليكتروني :E-Branch: بدأت بعض البنوك المصرية في الاتجاه إلى فتح فروع إليكترونية لتقديم خدماتها للعملاء على مدار الأربع والعثرين ساعة وفى غير مواعيد 
العمل الرسمية وذللك من خلال توفير عدد من الخدمات مثل ماكينات الصرف الآلي ، وكذا

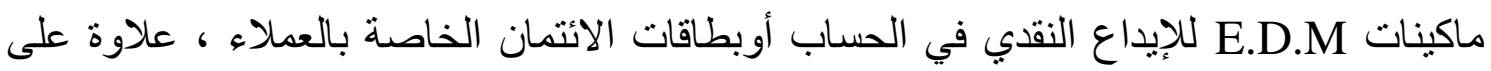
- Call Center توافر ال و- المواقع على شبكة الانترنت Web Site : هناك العديد من البنوك المصرية التي لها

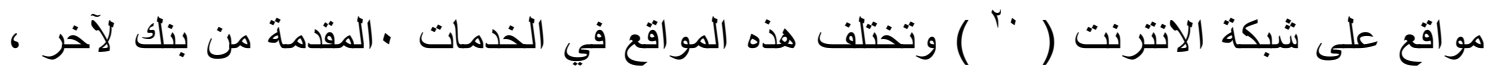
حيث هناك صفحات للبنوك تقتصر على عروض ميزانيات البنك ، وكذا عدد من الخدمات المقدمة

[ تختلف تللك الخدمات نوعاً وحجماً من بنك لآخر ] و الربط مع مواقع المصادر الرسمية للبيانات الاقتصادية و المصرفية بمصر ، إلا أن هناك بنوك تقدم من خلال رقم سرى خاص للعميل إمكانية

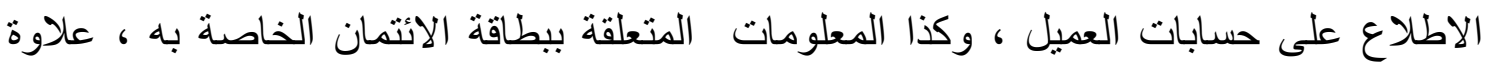

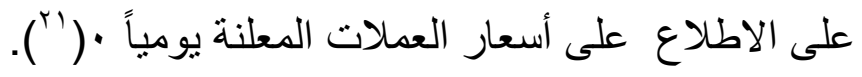

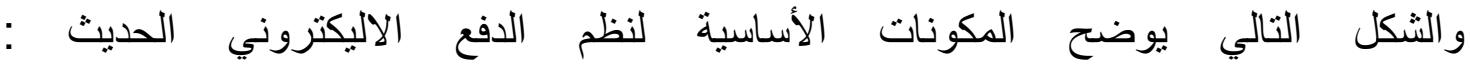
Swift

Automatic
clearing System

E-commerce
Internet

Payment

\section{Credit card}

\section{Mobile Payment}

المصدر : إتحاد بنوك مصر ، مجلة البنوك ، العدد 010 ، 9 . . ، ص 7 ،

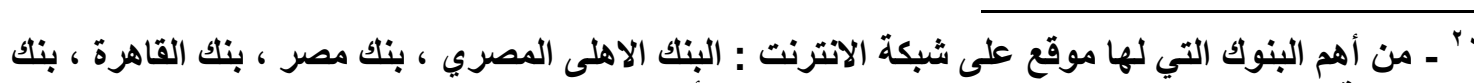

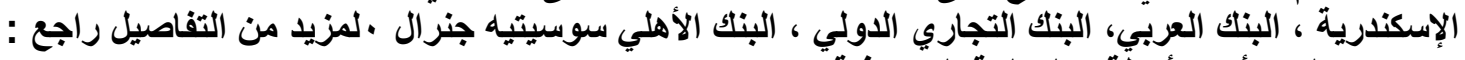

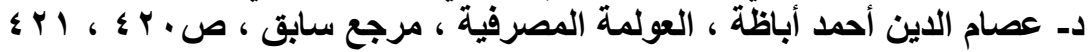

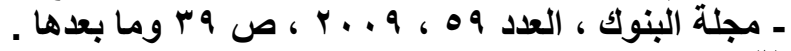

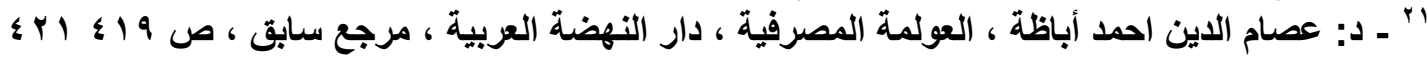




\section{ثنانياً : مخاطر الصيرفة الإليكترونية :}

تعرف المخاطر (rr ) بأنها احتمالية تعرض البنك إلى خسائر غير منوقعة وغير مخطط لها

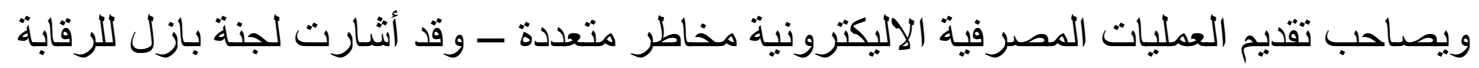
المصرفية إلى انه ينبغي أن تقوم البنوك بوضع السياسات و الإجراءات التي تتيح لها إدارة هذه

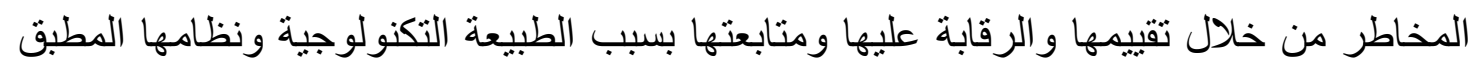

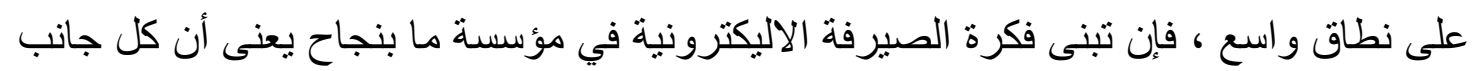

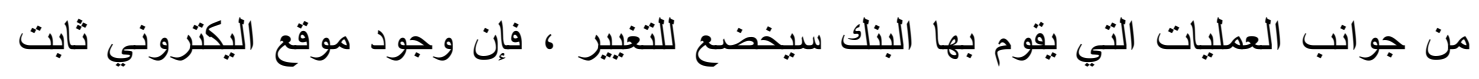

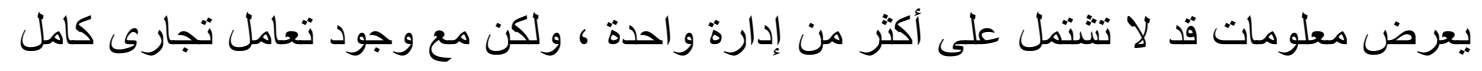
ونموذج لثركة صيرفة اليكترونية [ Corporate E- Banking ينطلب ذلك تكامل للأنظمة

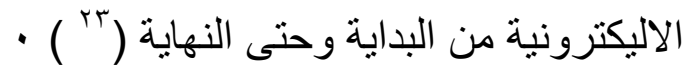

$$
\begin{aligned}
& \text { وفيما يلي عرض مفصل لهذه المخاطر( }
\end{aligned}
$$

ا- مخاطر التشغيل Operational Risk : ويمكن النظر للمخاطر التشغيلية بمعناها الواسع على أنها جميع المخاطر غير السوقية [ Non Market Risk ] م والمخاطر غير الائتمانية Non Credit Risk، وقد عرفت لجنة بازل مخاطر التشغيل بأنها الخسائر التى قد تنتشأ

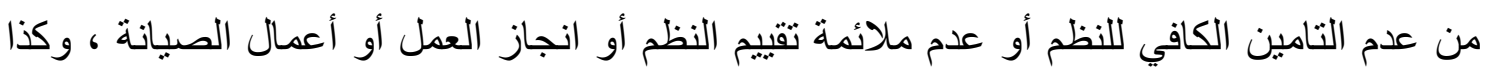

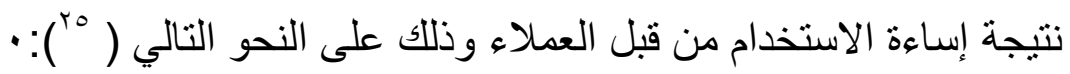

r r ـ يعرف الخطر Risk بأنه :حالة علدم التأكد المتعلقة بحصول الربح أو الخسارة ، كما يمكن تعريفه بأنه

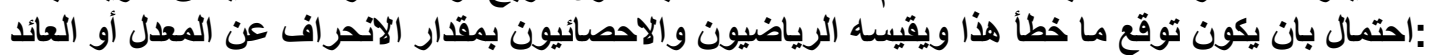

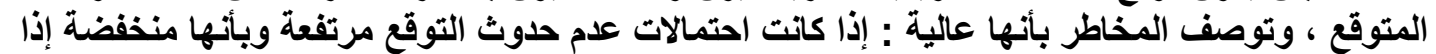

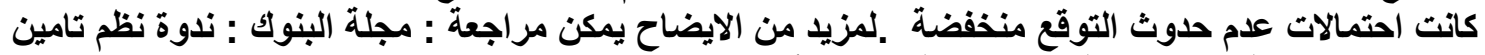

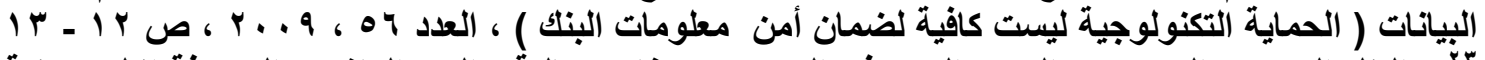

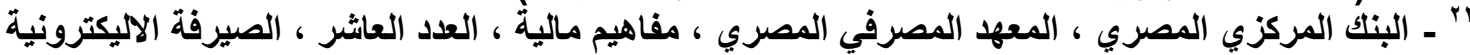

24- Alam and others, (2007)Development and Prospects of Internet Banking in Bangladesh , on International Business Journal Volume : 17, Issue : 1/2 . ( on - line ) Available on : www .emerald insight .com 15/5/2008 25-Basel-Committee on Banking Supervision. Anew Capital Adequacy Framework .Consultative Paper. Basel.June1999.pp.12-18

-BANK FOR International Settlements .Basel Committee on Banking Supervision .Consultative Document .The new Basel Capital Accord. January 2001 
// / عدم التامين الكافي للنظم System Security وتنشأ هذه الهخاطر عن إمكان اختراق غير المرخص لهم Unauthorized Access لنظم حسابات البنك بهدف التعرف على

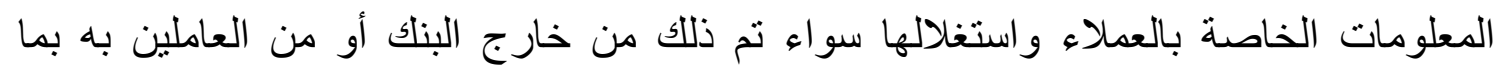
يستلزم تو افر إجر اءات كافية لكثف و إعاقة ذللك الاختراق .

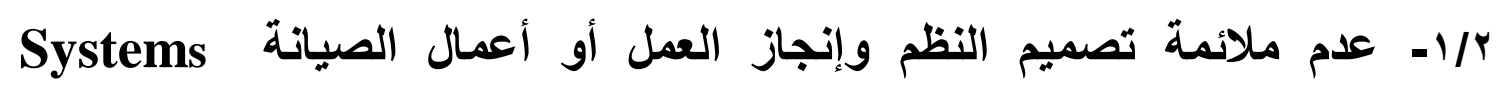
Design. Implementation. and Maintenance أو عدم كفاءتها [ بطء الإدارة Slow - Down على سبيل المثال ] لمواجهة متطلبات المستخدمين - وعدم السرعة في حل هذه المشاكل وصيانة النظم - وخاصة إذا زاد الاعتماد على مصادر خارج الإدان

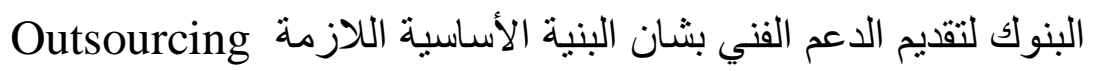
ب/ ا - إساءة الاستخدام من قبل العملاء Customer Misuse of Services ويرد ذلك الك نتيجة عدم إحاطة العملاء بإجراءات التامين الوقائية Security Precautions أو بسماحهم لعناصر إجرامية بالدخول إلى حسابات عملاء آخرين أو القيام بعمليات غسيل الأموال باستخدام

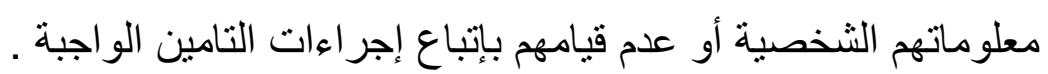
r- المخاطر القانونية Legal Risk : تمثل المخاطر القانونية التي قد تتشأ عن العمليات

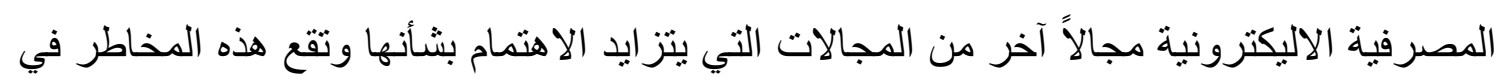

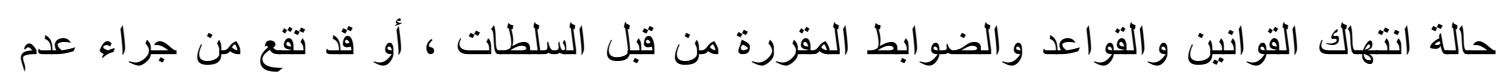

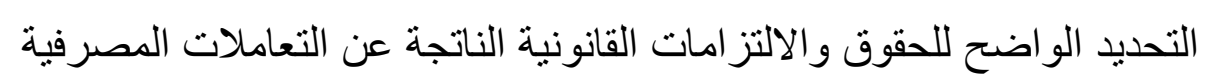

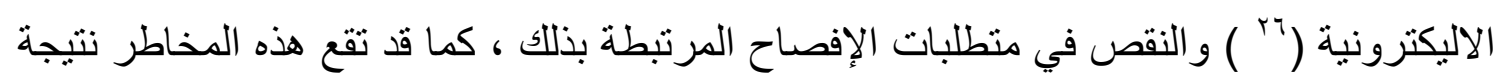

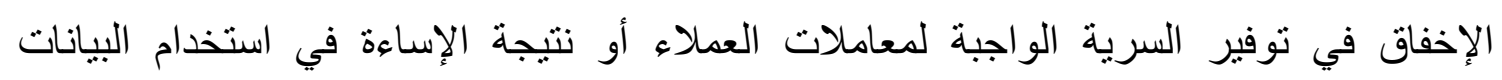

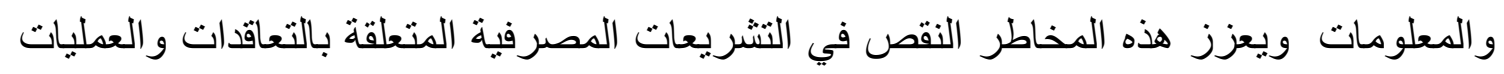
الاليكترونية والآليات القانونية لضبط تنفيذ هذه التعاقدات والتعاملات ـومن الأمثلة الثائعة لهذه

"r ـ ومن ذلك عدم وضوح مدى تو افر قو اعد لحماية المستهلكين في بعض الدول ، أو لعدم المعرفة القانونية

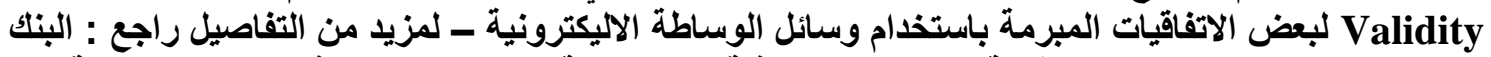

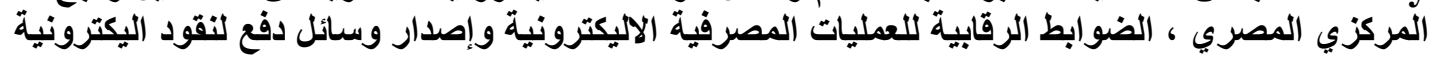


المخاطر قيام المصارف بتقديم خدمات مصرفية على شبكة الانترنيت لعملاء خارج النطاق القانوني أو القضائي لهذه المصارف كما هو الحال في العمليات عبر الحدود . r- مخاطر السمعة الايتمانية Reputational Risk يرتبط نجاح المصارف في أعمالها بالسمعة التي تؤسسها كمؤسسات جديرة بالثقة ، وتنشأ مخاطر السمعة فيما ينعلق

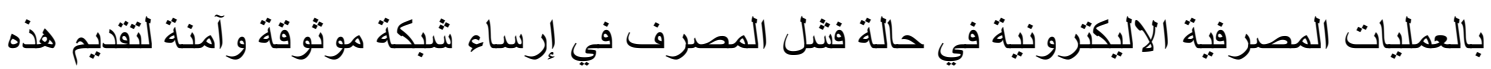

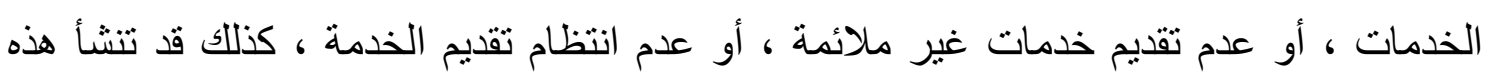

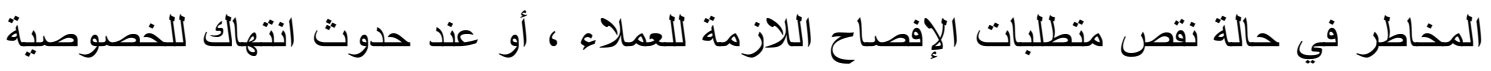

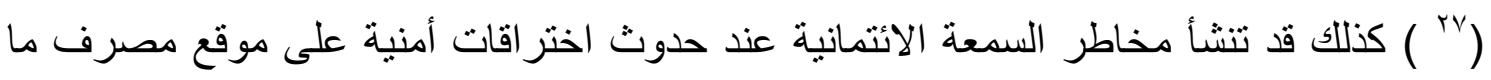
في شبكة الانترنيت وقد تمتد من جر اء ذلك هذه المخاطر لتشمل مصارف أخرى نتيجة فقدان ثقة العملاء أو السوق بمقرة المصارف بصورة عامة على الإدارة السليمة للمعاملات المصرفية الاليكترونية .

عـالمخاطر الأخرى :يرتبط أداء العمليات المصرفية الاليكترونية بالمخاطر الخاصة بالعمليات

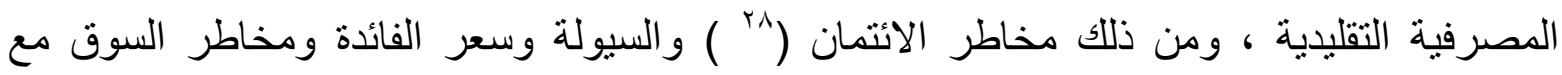
احتمال زيادة حدتها ، فعلى سبيل المثال فإن استخدام قنوات غير تقليدية للاتصال بالعملاء وامتداد نشاط منح الائتمان إلى عملاء عبر الحدود Cross - Border قد يزيد من احتمالات إخفاق بعض بهل

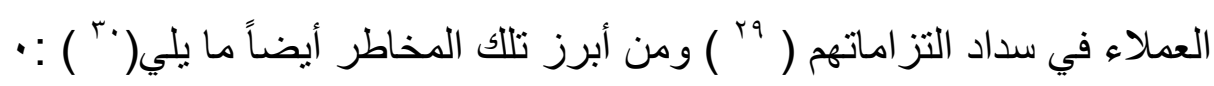
1/ء - اختراق الأنظمة : ويتحقق بدخول شخص غير مخول بذلك إلى نظام الكمبيوتر والقيام

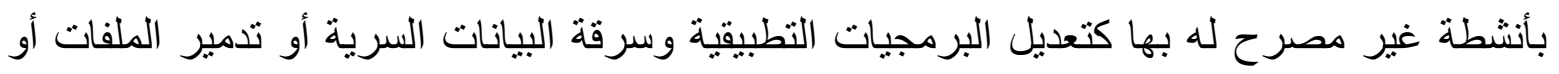
البرمجيات أو النظام أو لمجرد الاستخدام غير المشروع ، ويتحقق الاقتحام بشكل تقليدي من خلال

v ـ ـ البنك المركزي المصري الضوابط الرقابية للعمليات المصرفية الاليكترونية ، وإصدار وسائل دفع لنقود

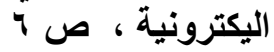

28-Anew Capital Adequacy Frame Work Consultative Paper .Issued by Basel Committee on Banking Supervision .June1999.

29-Basel Committee on Banking Supervision .Quantitative impact study. Technical Guidance .october2002.

ـ البنك المركزي المصري ، دليل التعليمات الرقابية الصادرة عن قطاع الرقابة والإثراف ، الباب الخامس ـ

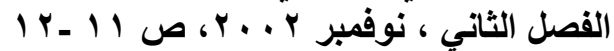

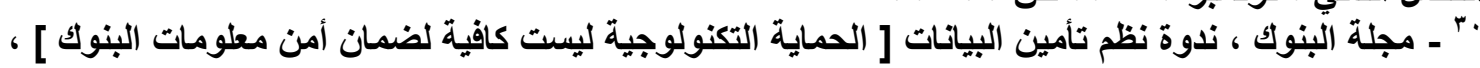


أنثطة التخفي وير اد به تظاهر النخص المخترق بأنه شخص آخر مصرح له بالدخول ، او من خلال

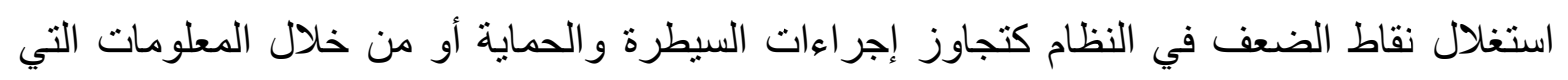

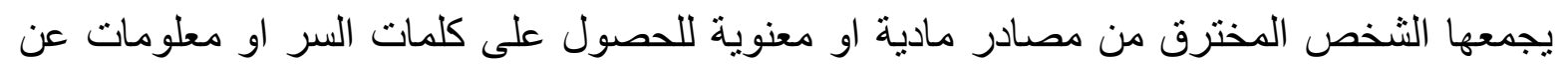
النظام او عن طريق الهندسة الاجتماعية كدخول الثخص إلى مواقع معلومات حساسة داخل النظام ككلمات السر أو المكالمات الهاتفية

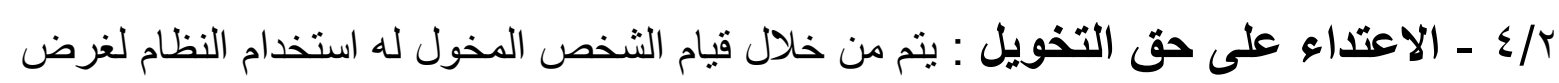
ما باستخدامه في غير هذا الغرض دون حق ، وهذا الخطر يعد من الأخطار الداخلية فى حقل إساءة

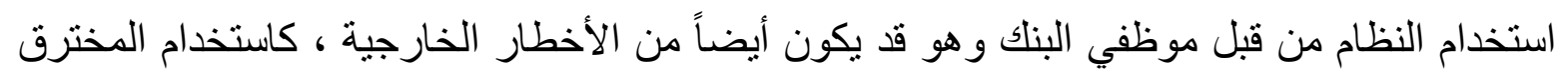

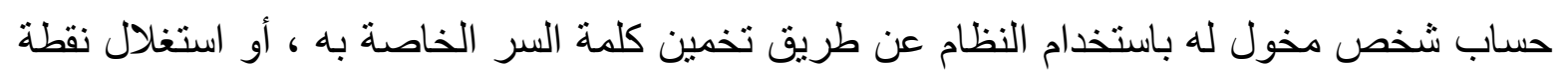

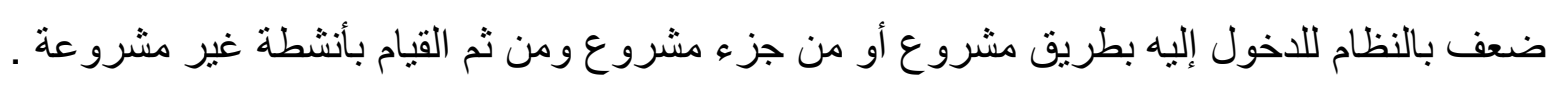

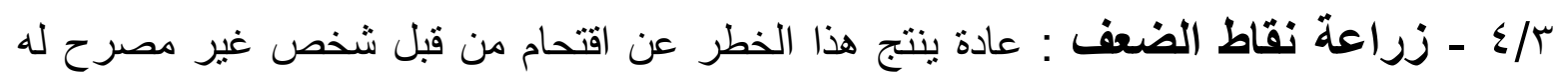
بذلك

أو ون خلال مستخدم مشروع تجاوز حدود التخويل الممنوح له بحيث يقوم الشخص بزرع مدخل ما.

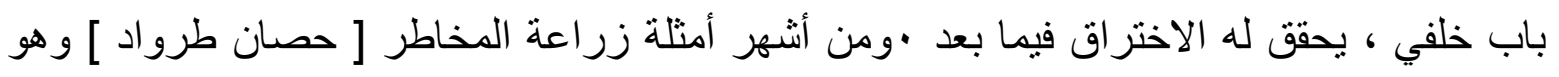

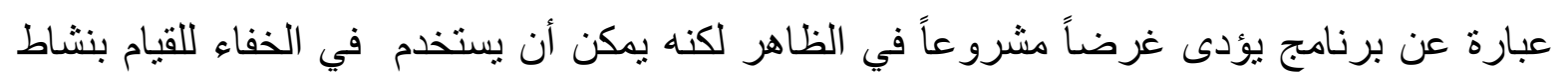

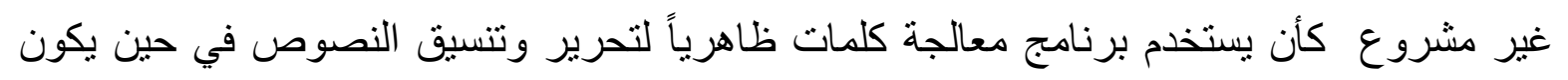
غرضه الحقيقي طباعة كافة ملفات النظام ونقلها إلى ملف مخفي بحيث يمكن للمخترق أن يقوم بطباعة هذا الملف و الحصول على محتويات النظام •

ع/عـ - مراقبة الاتصالات : بدون اختراق كمبيوتر موظف البنك يتمكن القرصان Hecker من

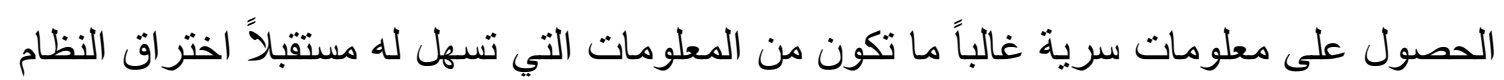

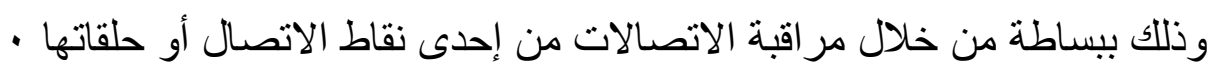

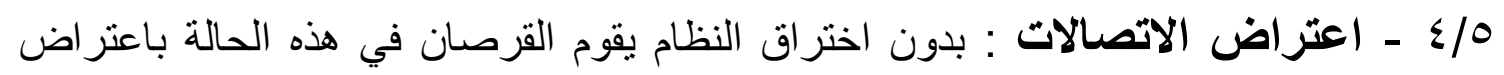

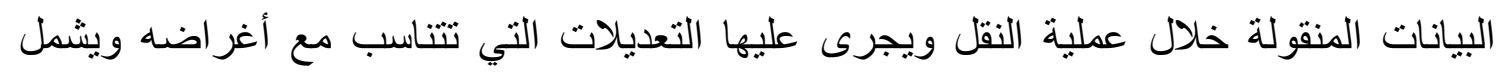
اعتراض الاتصالات فيام القرصان بخلق نظام وسيط وهمي بحيث يكون على المستخدم أن يمر ولئ

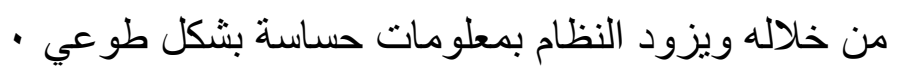


1/ ـ - تعطل الخدمة : ينم من خلال القيام بأنشطة تمنع المستخدم الثرعي من الوصول إلى

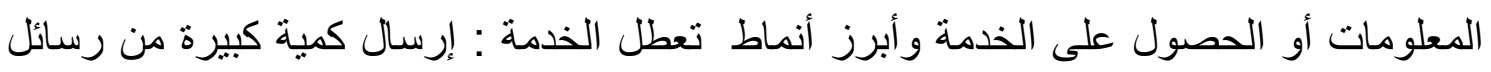

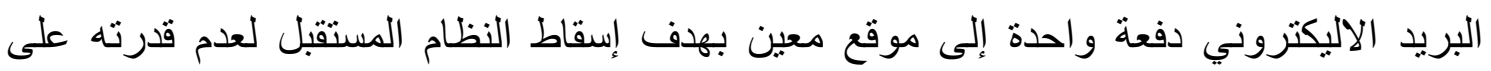

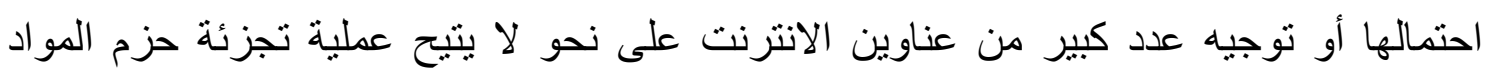

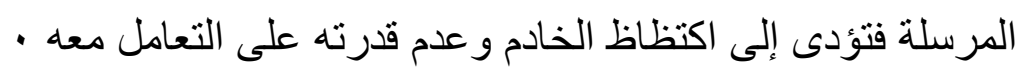
ع/V أو المرسل بالتصرف الذي صدر عنه ، كأن ينكر أنه ليس هو شخصياً الذي قام بإرسال طلب

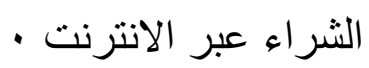

\section{ثالثاً :مدى إمكانية تحقيق الربحية في ظل مخاطر الصيرفة الإليكترونية :} يعتبر موضوع الربحية في المصارف من المواضيع الهامة في مجال الرقابة والتفتش على لهي المصارف وهو أحد أهم عناصر تقييم الوضع المالي للمصارف ويتأثر مستوى الربحية مبانشرة بمستوى كفاءة الإدارة ودرجة منانة ودقة نظام الضبط الداخلي ، فالإستر اتيجية المنتجة الفعالة

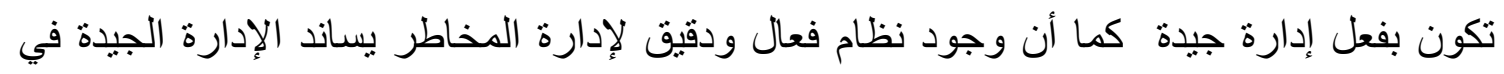

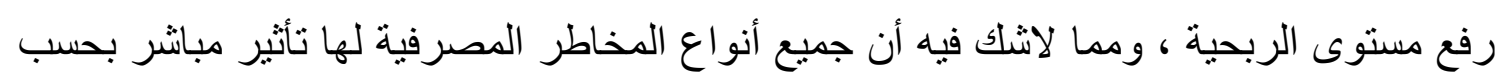

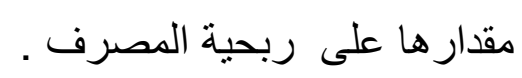
ولعل الإفصاح الاقيق والكافي عن مؤشرات تأثير المخاطر المعنية على ربحية المصرف هو

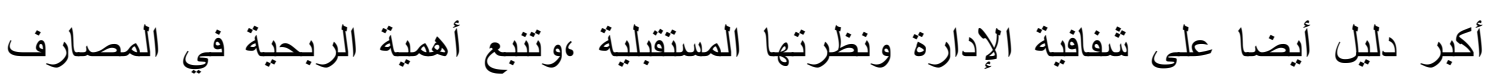

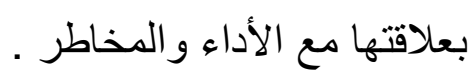

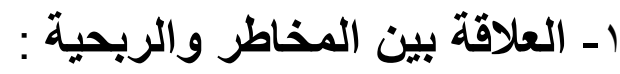

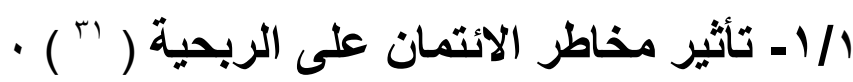

طبقت في بعض المصارف الكبرى في العالم منذ ما يزيد عن عقد من الزمان نماذج مقاربة

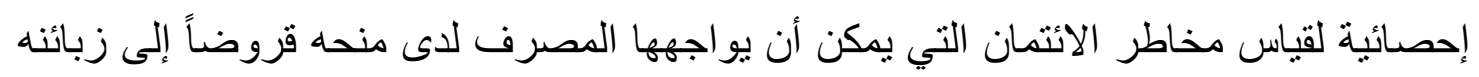

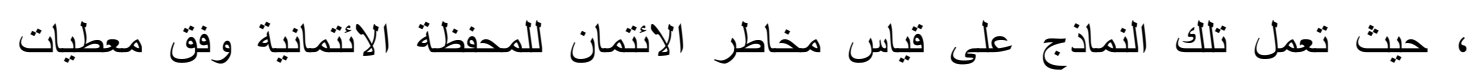


و افتراضات معينة يحددها المصرف المعنى سلفاً من واقع تعاملاته السابقة ، ويهدف هذا الأمر

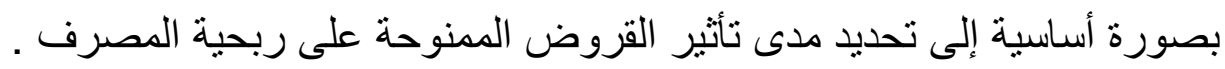

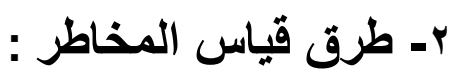

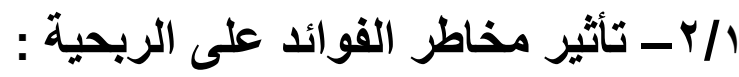

تقبس مخاطر الفائدة في الدرجة الأولى مدى تأثر أرباح المصرف في المدى القصير ومدى تأثر

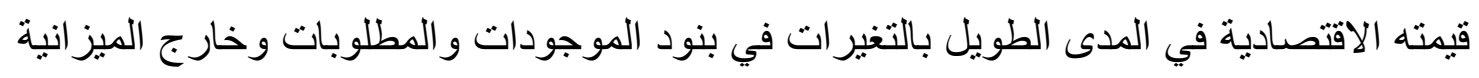

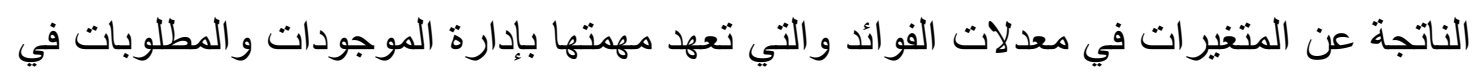
المصرف .

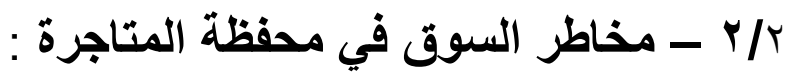

تعمل المصارف الدولية على قياس أنواع محددة من مخاطر السوق ، وكنلك مخاطر الإقراض

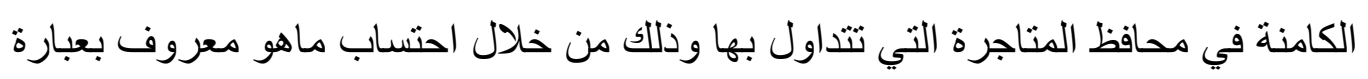

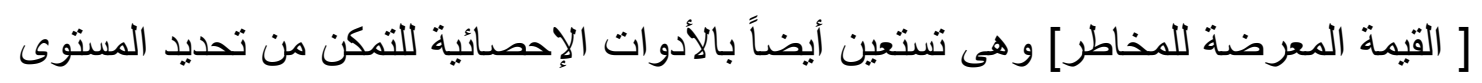
من الخسائر الناتجة عن المخاطر التي يتوقع تجاوزها في المراكز المحتفظ بها حالياً وذلك خلال فترة زمنية محددة, rـ نسب الربحية Profitability Ratios يجد المعنيون والمتمون بأداء المصارف في الدول التي لا تتمتع عموماً بأسواق مالية منظمة ونشطة وذات سيولة عالية فى نسب الربحية ،

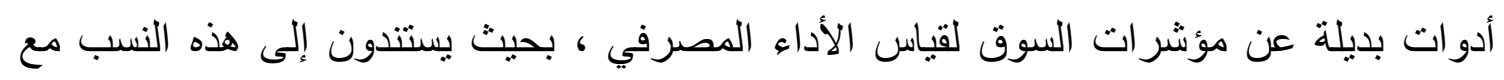

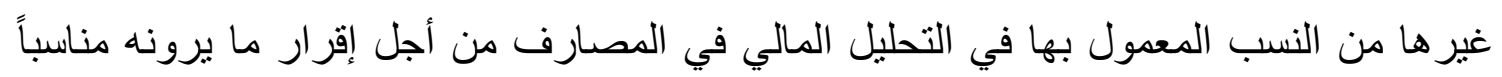
في تعاملهم مع المصرف المعنى وفيما يلي أهم مكونات هذه المجموعة من النسب :

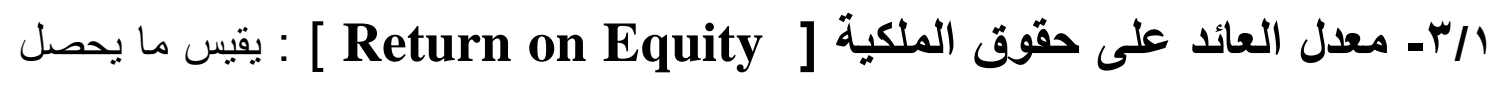

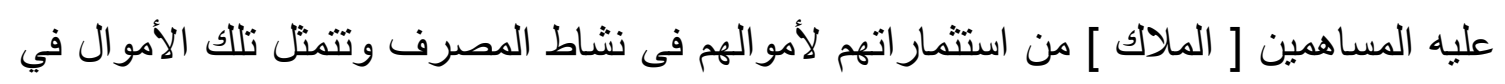

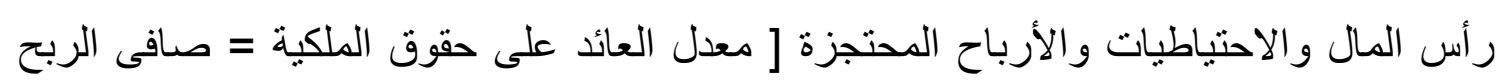

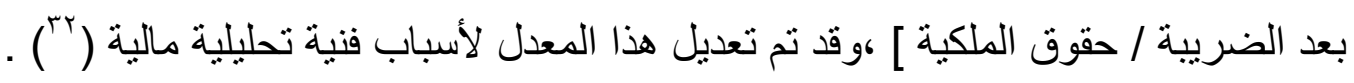

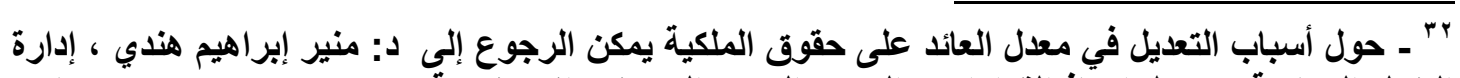

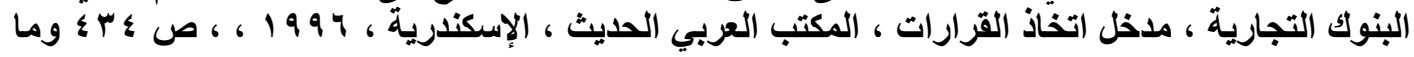


معدل العائد على الملكية = صافى الربح بعد الضريبة / حقوق الملكية + الأرباح المحتجزة + نصف الأرباح المتولدة عن السنة . ץ/ - معدل العائد على الودائع : يقيس مدى قدرة المصرف على توليد الأرباح من الودائع التي تم الحصول عليها : معدل العائد على الودائع = صافى الربح بعد الضريبة / الودائع بأنو اعها

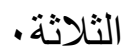

r/ץ- معدل العائد على الأموال المتاحة : يقيس نسبة صافى الأرباح المتولدة إلى جملة الموارد المتاحة المتمثلة في الودائع وحقوق الملكية Return on Total Resources معدل العائد على الموارد المتاحة = صافى الربح بعد الضريبة / حقوق الملكية +الودائع كessup .1980.p160 Net نسبة الفوائد المكتسبة إلى الفوائد المستحقة : يقترح interest Margin من الاستثمار ات : نسبة العوائد المكتسبة إلى الفوائد المدفوعة = الفوائد المكتسبة / الفوائد المستحقة

/ T- معدل العائد على الموارد المتاحة [ الاستثمار ] = صافى الربح بعد الضريبة / الخصوم + حقوق الملكية ، حيث أن الخصوم + حقوق الملكية تساوى إجمالي الأصول ، ويسود الاعتقاد بان المعدل المذكور يعد مقياساً لربحية الاستثمار ات (rّ") .التي تتكون من القروض و الأوراق المالية ،ويشير د/ منير هندي 1986 في الدراسة التي قام بها على هذا المعدل أن المقياس المذكور لم يعد مقياساً مقبو لاً لربحية الاستثمار ات ، كما أنه لا يخدم أي غرض آخر ؟/ اـ القوة الإيرادية للموارد المتاحة :إن عدم تو افر شرط التناسق وذلك إلى مكونات البسط [ صافى الربح بعد الضريبة ] في ظل نظام الضريبة التصاعدية قد يعطى المعدل السابق صورة مضللة عن ربحية الأمو ال المستثمرة . القوة الإيرادية للموارد المتاحة = صافى الربح قبل الضريبة + الفوائد المستحقة / الخصوم + حقوق الملكية 
ع ـ قواعد احتساب واستعمال نسب الربحية : تجدر الإشارة إلى أنه عندما يتعلق الأمر

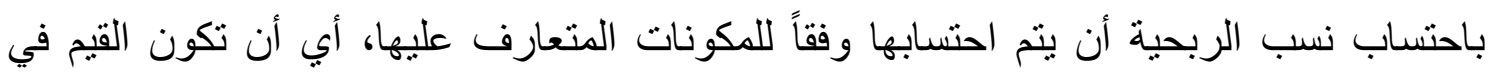
المقام على أساس منوسطات الأرصدة ، وان تكون هذه النسب قابلة للمقارنة ، و على سبيل المثال

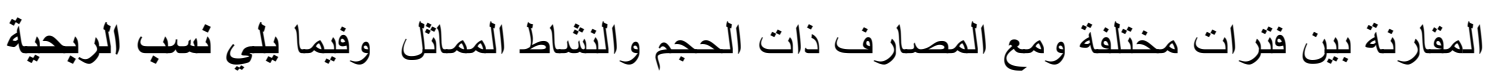
الرئيسية : صافى الأرباح

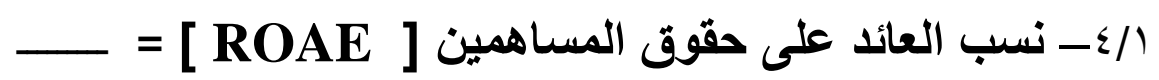
متوسط حقوق المساهمين

تقيس هذه النسبة معدل العائد للمساهمين ، أي معدل الربح الصافي الذي يجنيه المساهمون من

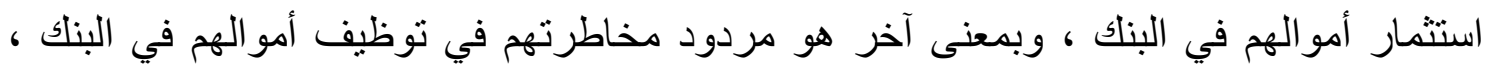
وتدل هذه النسب على مدى كفاءة إدارة المصرف في توظيف أمو ال المساهمين. صافى الأرباح

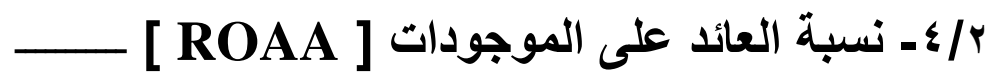
متوسط الموجودات

وتدل هذه النسبة على مدى كفاءة إدارة المصرف في استخدام وإدارة الموجودات وبالربط بين هاتين النسبتين يمكن الاستنتاج أن عائد البنك على حقوق المساهمين يتأثر بشكل كبير بكيفية تمويل

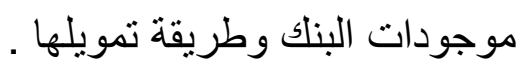

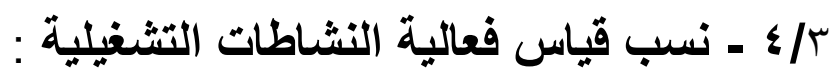
NET INTEREST ] [ نسبة صافى الهامش من الفوائد على منوسط الموجودات [ MARGIN الفو ائد المقبوضة ناقصاً الفو ائد المدفوعة

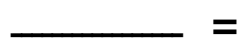
متوسط الموجودات المنتجة للفو ائد أو متوسط الموجودات تقيس هذه النسبة قدرة المصرف على تحقيق الأرباح من أنثطته الأساسية .

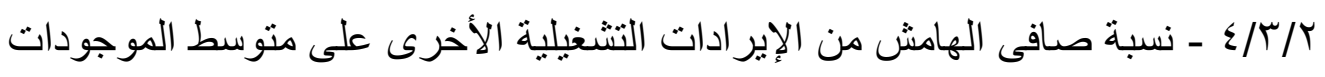


الإير ادات التشغيلية الأخرى ناقص الأعباء التشغيلية الأخرى

$$
\text { منوسط الموجودات }=
$$

تشير هذه النسبة إلى مدى نجاح البنك في تحقيق إير ادات من نشاطات غير تقليدية

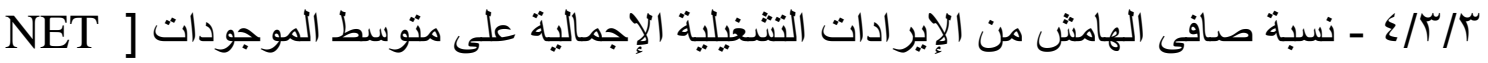

[ NON - INTEREST MARGIN

مجموع الإير ادات التشغيلية الإجمالية ناقص مجموع الأعباء التشغيلية الإجمالية

$$
\text { متوسط الموجودات }
$$

تدمج هذه النسبة النسبتين مع السابقتين I ، r لتعطى صورة متكاملة عن اداء المصرف على مستوى أنشطته التشغيلية الإجمالية .

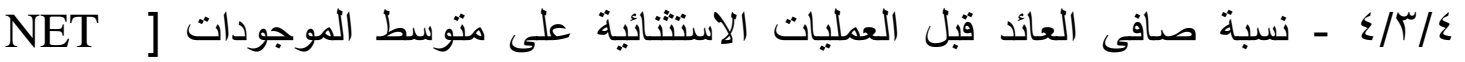
. [ N.R.S.T ] [RETURN BEFORE CPECIAL TRANSACTIONS صافى الأرباح بعد الضرائب وقبل الأرباح أو( الخسائر) من العمليات الاستثنائية

$$
\text { متوسط الموجودات }
$$

تقيس هذه النسبة أرباح البنك من مصـادر دخله العادية الإيرادات من التسليفات ، الاستثمارات و العمو لات من الخدمات المالية الأخرى ، الخ ....] إلى مجموع الموجودات المنتجة للإير ادات ولا تشمل العمليات غير العادية [ مثلاً بيع أملاك البنك أو تجهيزاته ، الخ ...] وتختلف هذه النسبة بالتالي عن نسبة الموجودات بكونها لا تلحظ فيها الأرباح أو الخسائر الاستثنائية والضرائب المدفو عة عليها

\section{التنائج و التّوصياث}

تناولت الدر اسة بالبحث و التحليل موضوع الخدمات المصرفية الاليكترونية وقد تبين منها : ا-إن التغير السريع في تطبيقات تكنولوجيا المعلومات والاتصالات أدى إلى أن العالم أصبح يتغير كل يوم وبوتيرة متسار عة ، وفى مصر التي اختارت أن تكون جزءاً من النظام الاقتصادي 
العالمي المفتوح فأصبحت البيئة المصرفية تتأثر بتلك التقلبات المتسارعة التي تتعرض لها الاقتصاديات العالمية ، وبالتالي أصبحت البيئة الاقتصادية والمصرفية في مصر بيئة متغيرة يتحتم التعامل معها بمفاهيم جديدة تلائم التقلبات التي تحدث في بيئة الأعمال المصرفية . r-تطرقت الدر اسة إلى بيان مفهوم الخدمات المصرفية الاليكترونية والذي يتمثل فى : تقديم البنوك مكي الخدمات المصرفية التقليدية أو المبتكرة من خلال شبكات اتصال اليكترونية تقتصر صلاحية الاخول إليها على المشاركين فيها وفقاً لشروط العضوية التي تحددها البنوك وذلك من خلال أحد المنافذ على الثبكة كوسيلة لاتصال العملاء بها - التها r-أبانت الدراسة عن تتوع وتعدد الخدمات المصرفية الاليكترونية التي تقدمها البنوك في الوقت الحاضر ومنها : بطاقات الدفع الاليكترونية بنوعيها [ بطاقات الخصم - وبطاقات الائتمان ] ، و الخدمات المصرفية عن بعد ، خدمات الصيرفة المنزلية ، الخدمات المصرفية عبر شبكة الانترنت ع-ثم تطرقت الدراسة أيضاً لبيان دور البنوك المصرية في مجال نشر قنوات التوزيع الاليكترونية ومن أهمها : نقاط البيع الاليكثرونية ، آلات الصارف الآلي ، البنك الثخصي [ الصيرفة

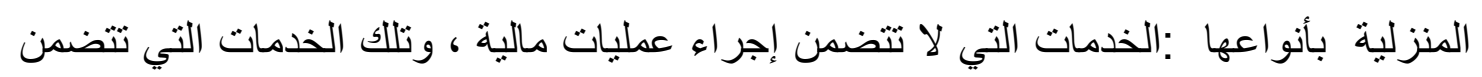
إجراء عمليات مالية ، ثم تطبيق نظام البنك المحمول ، وما يعرف بالفرع الاليكتروني حيث بدأت بعض البنوك المصرية في الاتجاه نحو فتح فروع اليكترونية لتقديم خدماتها للعملاء على مدار الأربع والعثرين ساعة وفى غير مواعيد العمل الرسمية ، وكذا المواقع على شبكة الانترنت

هـأكدت الدراسة على المخاطر العديدة للصيرفة الاليكترونية والتي تمثلت في:المخاطر التشغيلية بسبب ] عدم التأمين الكافي للنظم ،أو عدم ملائمة تصميم النظم وانجاز العمل أو أعمال الصبانة أو إساءة الاستخدام من قبل العملاء ] ،المخاطر القانونية ، ومخاطر السمعة الائتمانية ،وطائفة من المخاطر الأخرى التي تصاحب أداء العمليات المصرفية الاليكترونية . 7-ثم تطرقت الدراسة لبيان إمكانية تحقيق الربحية في ظل مخاطر الصيرفة الاليكترونية ، حيث كثفت الدراسة عن حقيقة جوهرية هامة هي أن هناك علاقة وثيقة بين مخاطر الصيرفة الاليكترونية والربحية التي تبغي المصارف تحقيقها من جراء قيامها بتلك الخدمات وان هناك هي 
تأثير سلبي لتلك المخاطر على تلاك الربحية وأن المصارف الكبرى في العالم قد طبقت منذ ما

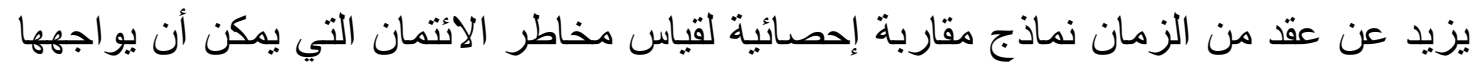

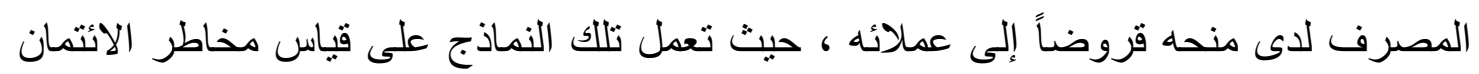

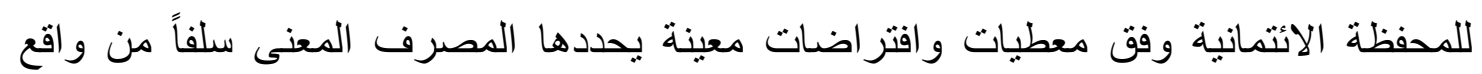

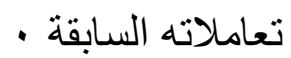

V-أظهرت الدراسة حقيقة جوهرية هي أن : حساب النتائج يعكس الوضع المالي للمصرف حيث أن

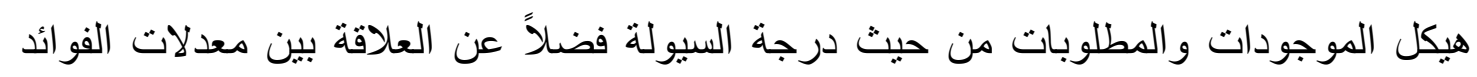
المختلفة تحدد صافى الإيرادات من الفوائد ، كما وأن تركيبة توزيع الودائع تحتم اختيار

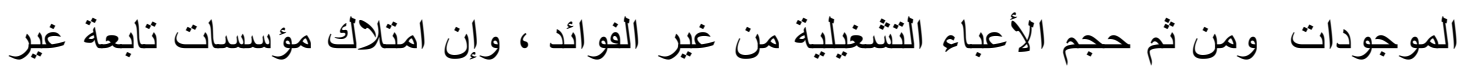

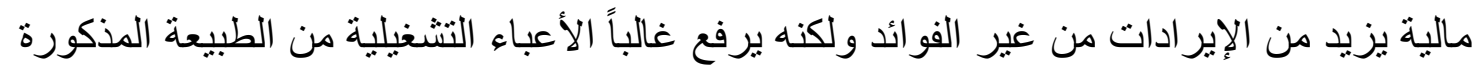

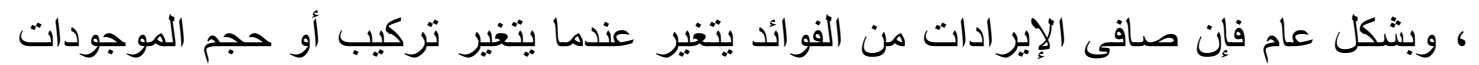

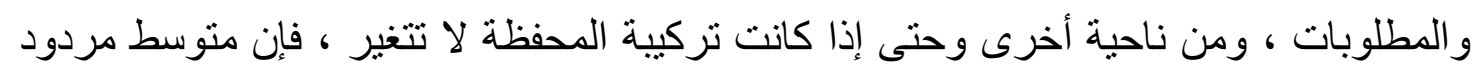

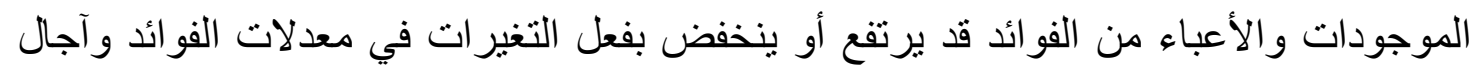
الأدوات المعنية ، و هذه التركيبة في الميز انية تنعكس في حساب النتائج على الإير ادات و والأعباء

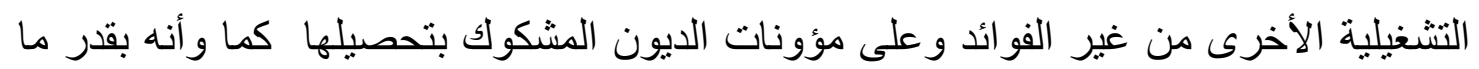
يكبر حجم تسليفات المصرف يكبر معها حجم النفقات العامة وحجم المؤونات ^ـ اهتمت الدراسة ببيان أهم مكونات نسب الربحية في المصارف و التي تمثلت في : معدل العائد

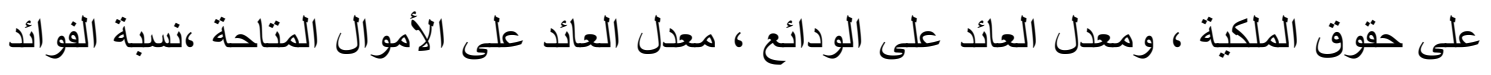

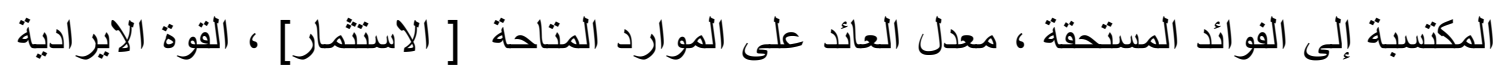

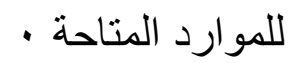
9- ثم تطرقت الدراسة بالتحليل لقو اعد احتساب واستعمال نسب الربحية في المصارف وأبانت عن تعدد وتتوع هذه القو اعد مثل : نسب العائد على حقوق المساهمين ، نسبة العائد على الموجودات 
ثانياً التوصيات : ومن هذه الدراسة يمكن تقديم التوصيات الآتية :

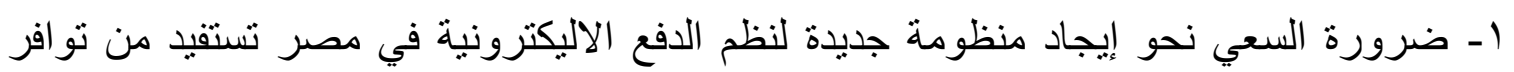

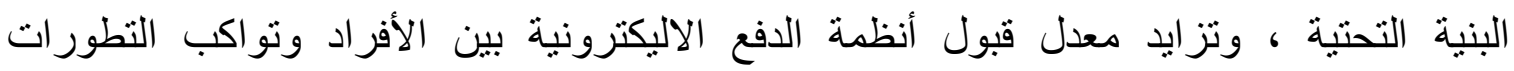

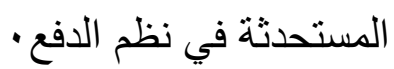
r- يجب الأخذ في الاعتبار تأثثر الآليات المستحدثة على منظومة الدفع القومية التي تسعى البنوك المركزية في مختلف البلدان إلى العمل على تطوير ها ، ويجب العمل على إعادة بناء نظم الدفع بعد

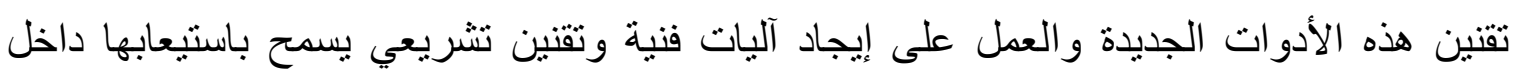
النظام حتى لا تتسرب عمليات الدفع إلى خارج منظومة الدفع القومية ، ويفقد النظام المصرفي جانباً

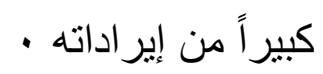
r-على الرغم من أن سوق نظم الدفع في مصر ماز ال يعانى العديد من المعوقات إلا إنه استناداً لعدد

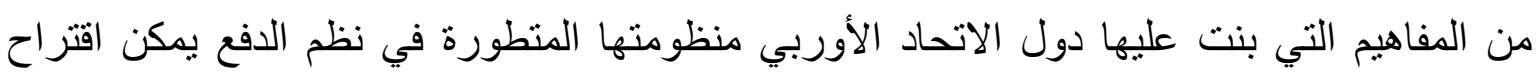

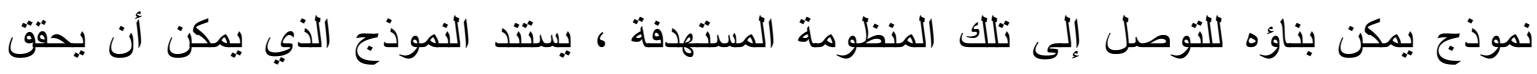
المنظومة الجديدة في نظم الدفع إلى العمل على تجميع كافة نظم الدفع في نسق واحد بحيث يضم

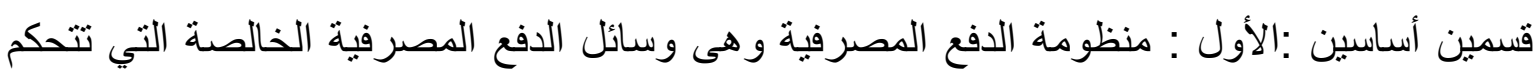

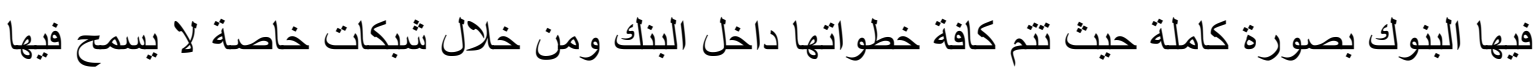
لأي مؤسسة غير مصرفية بالمشاركة فيها وتضم المنظومة نظام السويفت وبطاقات الائتمان

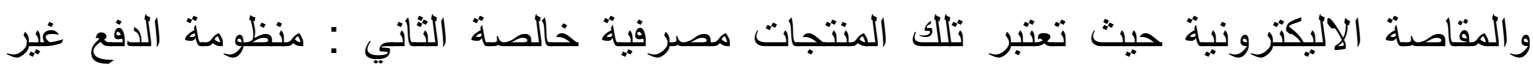

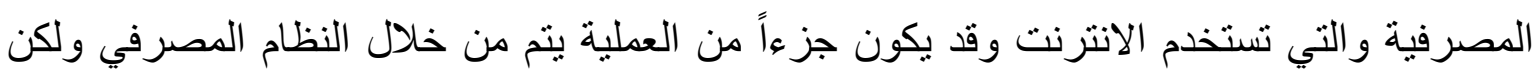
الأطراف الأخرى تثارك فيها بصورة أساسية فعلى سبيل المثال في خدمة Mobile Payment يعتبر ال Mobile operator هو اللاعب الرئيسي في المنظومة وفى خدمة ال Internet Payment تعد المنظمات الافتر اضية اللاعب الرئيسي ، و العديد من النظم لا تستخدم النقود العادية في تعاملاتها و إنما تستخدم النقود الاليكترونية ، والعديد من نظم الدفع القومية تتجاهل أثناء تصميمها هذه النظم الجديدة والتي يطلق عليها خدمات وساطة المدفوعات والتي تمنلك قدرات تنافسية يمكن بها أن تزيح البنوك عن عروشها التقليدية في عالم الددفوعات إذا اسنطاعت تحقيق قدر من المصداقية حيث أنها

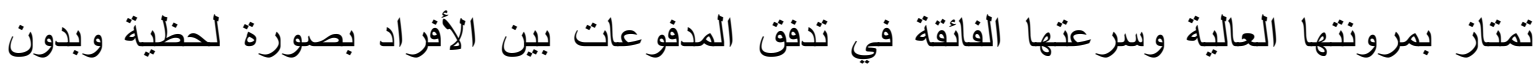


تعقيدات إدارية وبتكلفة منخفضة للغاية مما يجعل هذه التنظيمات مرشحة لكي تلعب دوراً هاماً في نظم الافع الاليكثرونية في الأجل القصبر عــهالك العديد دن العناصر المهمة التي تحدد هيكل بنوك الغد ومنظومة الخدمات التي ستقدمها البنوك لعملائها في المستقبل ، و على الرغم من أن ضغوط المنافسة وطموحات الإدارة في الحصول على حصة سوقية مثلت في الماضي أهم العناصر التي حددت تطور البنوك في سوق الخدمات المصرفية ، إلا أن التطور المستقبلي للبنوك أصبح حالياً يحدده عنصر آخر هو القدر المتاح من التكنولوجيا المصرفية وتكنولوجيا الاتصالات التي أصبحت تلعب الدور الحاسم في رسم شكل البنوك ومنظومة الخدمات المصرفية في بنوك الغد • هـ في ظل التغيير القادم وسعى البنوك الدائم إلى تطوير آلياتها وأدواتها حتى تتواكب مع تلك المتغيرات سنشهد في خلال الأعو ام القادمة تحديات المنافسة في ميدان البنوك الاليكترونية بعداً جديداً يتمثل في انطلاق الجيل الثاني من البنوك الاليكترونية حيث ستكون هناك مؤسسات مالية تقدم على الثبكة خدمات كانت حكراً على البنوك بمعناها التقليدي أو بمعناها المقرر في تشريعات تتظيم العمل المصرفي ، وليس غريباً أن نجد مؤسسات تجارية أو مؤسسات تسويقية تمارس أعمالاً مصرفية بحتة نتجت عن قدر اتها المتميزة في إدارة موقع مالي على الثبكة ، وأصبحت بنكاً حقيقياً بالمعنى المعروف بعد أن كانت تعتمد عبر خطوط مرتبطة بها على البنوك القائمة . 7-إن تطور المعاملات الاليكترونية وانطلاقها لا يمكن أن يكتب له النجاح المنشود إلا في وجود بيئة تشريعية ملائمة تساير - إن لم تكن تسبق -التطور المتسارع ى هذا الاتجاه وقد وضعت لجنة بازل للرقابة المصرفية العديد من الخطوط العريضة التي يجب على البنوك التي تتعامل في هذا الاتجاه أن تتبناها ، و لاشك أن تهيئة البيئة التشريعية والتظيمية بالصورة الملائمة ، مع التوسع في نشر الثقافة التكنولوجية و المصرفية في المجتمع المصري ، وفى ظل وجود كوادر بثرية مدربة ، سوف يساهم في إعطاء قوى الدفع الملائمة للخدمات المصرفية الاليكترونية والتوسع فيها خلال المرحلة القريبة القادمة لذا فنرى : - ضرورة دراسة وتقييم كل منتج على حدة لمعرفة جدوى التوسع فيه مستقبلاً على مبدأ التكلفة و العائد ، حتى يكون هنالك مردود إيجابي للمنتجات المزمع إدخالها مستقبلاً - ضرورة تدعيم الجهات الرقابية بالكوادر والكفاءات اللازمة لمتابعة أعمال وخدمات البنوك الاليكترونية نظر اً لحداثة التعامل بتلك الخدمات ـ نسبياً في السوق المصري . 


\section{ABSTRACT}

In the light of the economic challenges incurred by the century winds, including globalization, knowledge, and the major economic blocs among many countries of the world, had to be accompanied with financial services with the developments of the times, so that they can be rapidly and accurately at the international level, where the tools of advanced technology has entered more intensive in the banking sector in the prior period and has proved efficient and highly efficient in performance and the compability in banking services and largely reflected in the growth of electronic means of payment and development of banking services and effectively.

Application of knowledge and electronic fields which accused by number of Egyptian banks and which is expected to expand to deal with the future in the light of rapid advanced witnessed in the information technology industry in Egypt and the growing numbers of internet users in general on the other hand.

There is no doubt that the application of banking electronic systems requires the availability of many of the basic pillars of either basic such as providing basic information and communication at the human level which included appropriate and qualified rehabilitation adequate as well as to support the cognitive awareness to the community, and educating customers the benefits that could accrue to them by using such new services. 


\section{المراجع العربية والأجنبية}

1-Bernardo Batiz -Lazo ,A historical Appraisal of information

Technology in commercial Banking University Business School,

$$
\text { July, } 2002, \text { p2 }
$$

-Richard Li Hua, (2007)Bench marking china firm competitiveness :a strategic framework, journal of technology management in china, volume :2,issue:2.(on- line) available on:www.emeraldinsight.com , 21/4/2008.

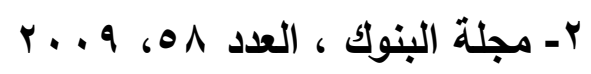

3- Awad Elias, (2004) Electronic Commerce: From Vision to Fulfillment, Pearson Prentice Hall, Upper Saddle River New Jersey.

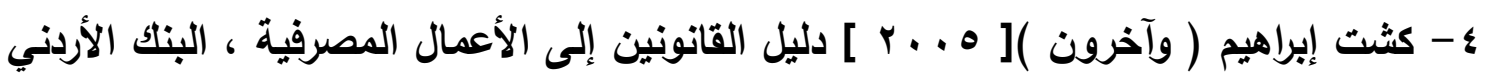

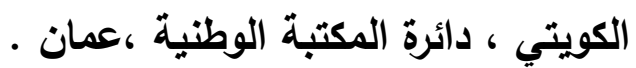

5-Electronic cash : The Funds or value is stored on an electronic device as the personal computer of the consumer Which is loaded using specialized software EC is used to Make small payments through a transfer of value to the merchants electronic device

6-Turban, E.lee\&, j.viehland, (2004),"Electronic commerce ". a managerial perspective, Pearson Education, Inc, upper saddle river, New jersey.

7-Kotler ,PH . and Keller , K.L ,(2006) Marketing Management "Pearson Prentice Hall Upper Saddle River,New Jersey

8- الأكاديمية العربية للعلوم المالية والمصرفية ، مجلة الدراسات المالية والمصرفية ، المجلد

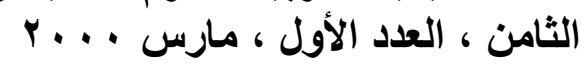

9-Kotler, ph.and Armstrong, G(2004)"principle of marketing "Pearson prentice Hall, upper saddle river, new jersey. 10--Karen Furst .William W.Lang.and Daniel E.Nolle. Internet Banking : Developments and Prospects.Economic And Policy Analysis Working Paper 2002 -9 September 2000.p16

11- Joseph ,P.T, (2004) E.commerce;;Amanagerial Perspective New Delhi "Prentice Hall of India ,Private Limited,

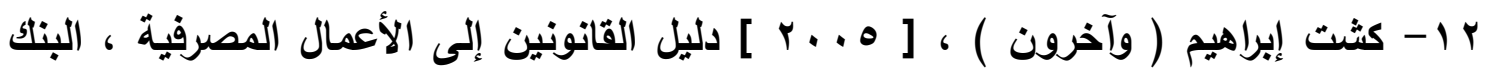

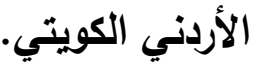




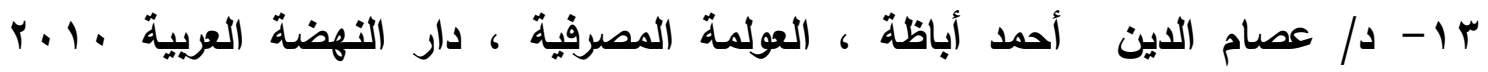
14-American Chamber of Commererce in Egypt Information Technology in Egypt , April 2002, p111- 112

15 - البنك الأهلي المصري ، النشرة الاقتصادية ، تطبيقات الصبرفة الاليكترونية في مصر العدد

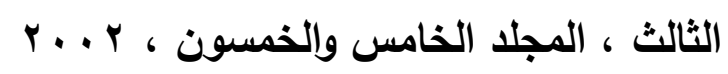

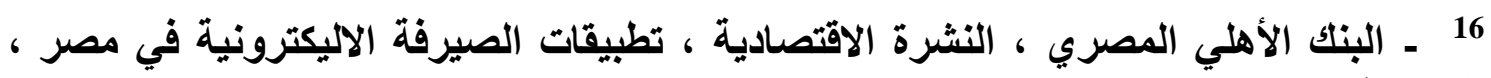

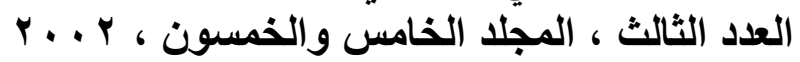

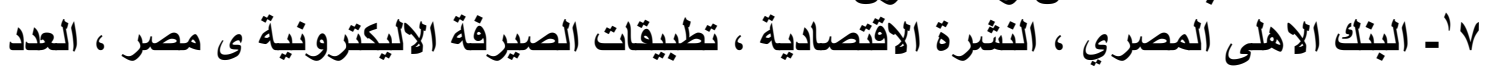

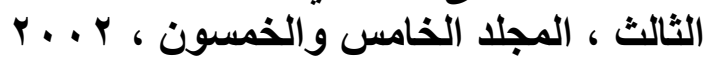
1 اــ البنك الاهلى المصري ، مركز البطاقات ، البنوك البون الاليكترونية وتطبيقاتها في مصر دراسة

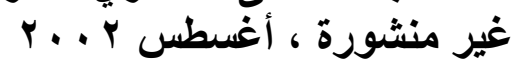

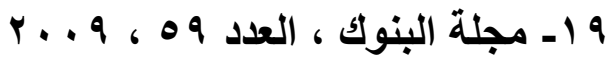

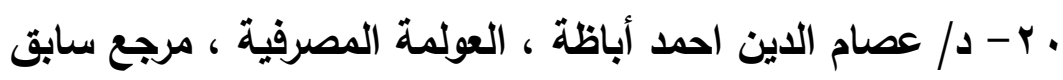

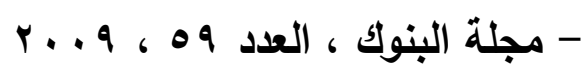

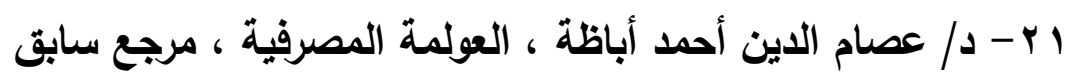

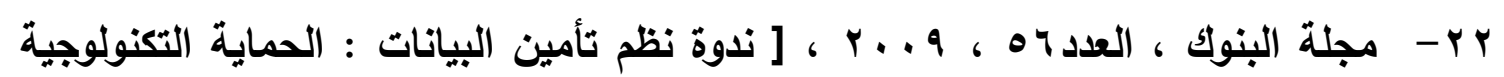

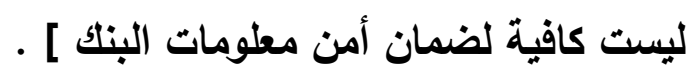

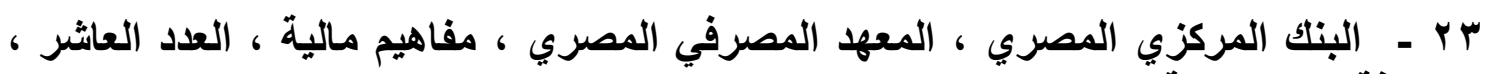

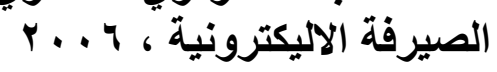

24- Alam and others, (2007)Development and Prospects of Internet Banking in Bangladesh, on International Business Journal Volume : 17, Issue : 1/2.( on - line ) Available on : www .emerald insight .com 15/5/2008 25-Basel-Committee on Banking Supervision. Anew Capital Adequacy Framework .Consultative Paper. Basel.June1999.pp.12-18 -BANK FOR International Settlements .Basel Committee on Banking Supervision .Consultative Document .The new Basel Capital Accord. January 2001

צr ـالبنك المركزي المصري ، الضوابط الرقابية للعمليات المصرفية الاليكترونية وإصدار

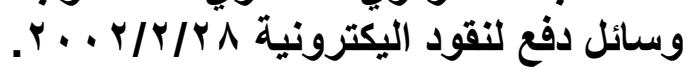

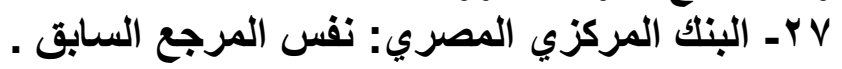
28-Anew Capital Adequacy Frame Work Consultative Paper .Issued by Basel Committee on Banking Supervision .June1999.

29- Basel Committee on Banking Supervision .Quantitative impact study. Technical Guidance .october 2002. 
- البنك المركزي المصري ، دليل التعليمات الرقابية الصادرة عن قطاع الرقابة والإشراف ،

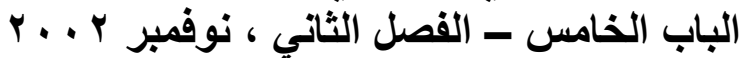
• بـ مجلة البنوك ، ندوة نظمّ تأمين البيانات [ الحماية التكنولوجية ليست كافية لضمان أمن

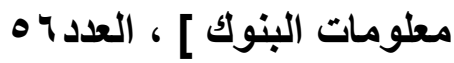
31- IMF .Finance \&Development. March 2001 -Financial-Times .Various issues -The Economist .Various issues rابـ د: منير إبراهيم هندي ، إدارة البنوك التجارية ، مدخل اتخاذ القرارات ، المكتب العربي

33-Gitman. 1993 .p131.Western\&

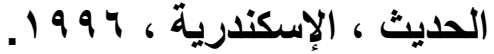

Brigham.1993.p132 www.eneraldinsight.com

-Sahay B.S \&others,(2006), managing supply chains for competitiveness :the Indian scenario. Supply chains management, An International journal, volume: 11, Issue: I(online) (Available on: www.emeral dinsight.com 21/4/2008. -Schullo, Reedy. (2004).E-commerce a managerial perspective, Pearson prentice Hall, Upper Saddle River, New Jersey. -Strauss j., Ansary A., Frost R.(2006),"E-Marketing". Pearson prentice Hall, upper saddle River, New Jersey. -Sekaran, Uma,(2002)"Research Methods for Business" fourth Edition, John Wiley\& sons,Inc. -Turban, E.lee, j.viehland, (2004),"Electronic commerce ". a managerial perspective, Pearson Education, Inc, upper saddle river, New jersey. -Winer, Russels (2004),"Marketing Management" New jersey: prentice Hall Englewood cliffs.p:40,232. -Mack, Tim, (2002): The growth of electronic marketing ": usa Today (magazine). 
7-Connor j \& glavin (2004) electronic marketing" theory \&practice for the21 st century, London ft prentice Hall. 8-Post Gerald \&Anderson David (2006) management in Formation system .New York mc grow ,Hill.

10-Sadig \& Shaikh \{2008\} Internet Banking and quality of service: Perspective From a developing Nation in the Middle East. On - line Information Review, Volume: 32, Issue: 1 \{on - line\} Available on:www. emera / dinsigh.com 13/05/2008.

11-Bernardo Batiz -Lazo ,A historical Appraisal of information Technology in commercial Banking University Business School, july 2002, p2 